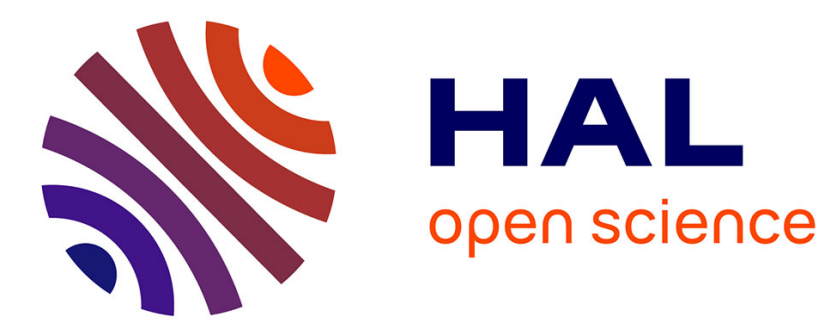

\title{
Lasers in astronomy
}

Renaud Foy, Jean-Paul Pique

\section{To cite this version:}

Renaud Foy, Jean-Paul Pique. Lasers in astronomy. Julian D C Jones and Colin E Webb. Handbook of Laser Technology and Applications, III (D8.1), Institute of Physics Publishing, pp.2581-2624, 2003, Applications, 978-0-7503-0607-2. 10.1887/0750306076/b1388v3c51 . hal-01119222

\section{HAL Id: hal-01119222 \\ https://hal.science/hal-01119222}

Submitted on 23 Feb 2015

HAL is a multi-disciplinary open access archive for the deposit and dissemination of scientific research documents, whether they are published or not. The documents may come from teaching and research institutions in France or abroad, or from public or private research centers.
L'archive ouverte pluridisciplinaire HAL, est destinée au dépôt et à la diffusion de documents scientifiques de niveau recherche, publiés ou non, émanant des établissements d'enseignement et de recherche français ou étrangers, des laboratoires publics ou privés. 


\title{
Lasers in Astronomy
}

\author{
Renaud Foy \\ Observatoire de Lyon /CRAL, France \\ Jean-Paul Pique \\ Laboratoire de Spectrométrie Physique, Grenoble, France
}

\section{Introduction}

This chapter addresses the problem astronomers are facing when using adaptive optics: there are not enough bright stars in the sky. In summary, the spatial resolution at the focus of astronomical telescopes is limited by the poor optical quality of Earth's atmosphere. It is due to turbulence, which is always and everywhere present, even at the quietest locations such as high altitude mountains where modern observatories are built. Adaptive optics is the most efficient tool for sharpening images to the ultimate diffraction limited resolution of the telescope. It requires relatively bright reference sources close to the programme object to measure phase disturbances on the incoming wavefront. Such sources are so scarce in the sky that the probability is very low that one will be close enough to the faint programme object, e.g. a quasar. The remedy to bright star scarcity is to create artificial ones close to the programme objects. It is achieved by pointing a laser beam in the sky at the target. Backscattering of the beam in the upper atmosphere, above the turbulent layers, can produce the required reference source. It is the so-called laser guide star (hereinafter referred as LGS).

In this chapter we will shortly introduce the formation of images through a turbulent medium and its consequences for imaging in astrophysics (Section 2).We will give the principle of adaptive optics and its limitations. It will lead us to the need for the LGS. We will review the three main backscattering process, addressing the laser matter interaction in the atmosphere (Section 3). Unfortunately, LGSs are not natural stars. They have physical properties which cause difficulties: they are at a finite distance, and they 
cannot be used to measure the tilt of the incoming wavefront over the full aperture of the telescope. These problems will be then discussed, as well as the way to overcome them, such as the polychromatic LGS (Section 4) and the arrays of LGSs to feed multiconjugate adaptive optics (Section 5), hereinafter referred as MCAO. The laser device requirements derived from the need to provide enough return photons, given the above mentioned problems are also solved (Subsection 2.3). We will discuss the most suitable laser devices to fit these requirements, depending on the solutions adopted (Section 6) and we will give examples of LGS devices installed at astronomical telescopes. Finally we will conclude by highlighting the main topics of research and development to bring the LGS concept into full operation at the largest telescopes in the world (Section 7).

\section{Image formation}

Images at the focus of telescopes are formed after propagation of the incoming wavefronts through a turbulent medium : the Earth's atmosphere. Let us summarize the optical properties of this medium. It will lead us to the conclusion that a laser guide star is required to get astronomical diffraction limited images for all observing directions in the sky.

\section{$2.1 \quad$ Spatial properties}

Due to turbulent mixing of convective flow, the temperature of Earth's atmosphere is not homogeneous at small spatial scales. In the free atmosphere, i.e.: above the ground layer, approximately $600 \mathrm{~m}$ thick, the temperature typically varies from $\lesssim 0.05^{\circ} \mathrm{C}$ at low altitudes to $\lesssim 0.001^{\circ} \mathrm{C}$ at an altitude of $3000 \mathrm{~m}$, between points separated by 1 meter above the best astronomical sites. Turbulence is located in a relatively few number of thin layers(e.g. Fig.1 (Vernin \& Mũnoz-Tuñón 1992)).

Temperature fluctuations cause variations in the refractive index of air, $n$. The fluctuations are Gaussian to a good approximation. Consequently the optical path length differs for two points on the incoming wavefront $W(\vec{r})$, causing phase delays; $\vec{r}$ is a two-dimensional (2-D) vector. For a point source at infinity, the atmospherically distorted wavefront surface entering the telescope can be written as: 

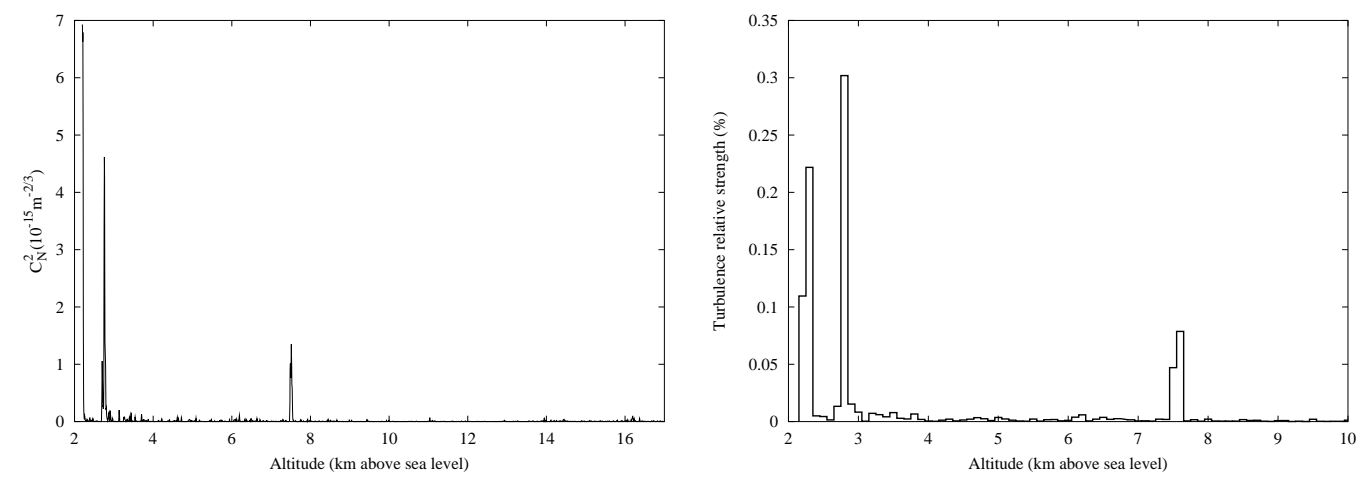

Figure 1: Distribution of $C_{N}^{2}$ with altitude on July 1991 above the Observatorio del Roque de los Muchachos, located at 2200m above sea level at La Palma island (Vernin \& Mũnoz-Tuñón 1992) Left: absolute profile. Right turbulence relative strength vertical profile. At other nights, at other sites, altitudes and relative strengths of the thin turbulent layers can be different, e.g.: frequently with peaks around $10 \mathrm{~km}$.

$$
A(\vec{r})=|\vec{r}| \exp \left(\frac{2 \pi i}{\lambda} W(\vec{r})\right)=|\vec{r}| \exp (i \phi(\vec{r}))
$$

where:

$$
\phi(\vec{r})=\frac{2 \pi}{\lambda} W(\vec{r})=\frac{2 \pi}{\lambda} n \Delta z,
$$

is the phase, and follows Gaussian statistics. $\Delta z$ is the length of the path through the (turbulent) atmosphere.

When the telescope mirror focuses the wavefront to form the source image, interferences are produced between coherent areas of the wavefront: the image of a point source shows a speckle pattern. The size of bright spots, equivalent to the fringe spacing in a 1-dimensional interferogramme, is determined by the largest baseline involved in the process, which is the telescope diameter $D$. Thus information is present in the speckled image down to the reciprocal telescope cutoff frequency $D / \lambda$ at the wavelength $\lambda$. This property of images formed through the turbulent atmosphere is the basis of speckle interferometry (Labeyrie 1970), of which the purpose is to restore diffraction limited images by postprocessing data obtained at the telescope.

The full width at half maximum (FWHM) of the image, of the whole speckle pattern, depends on the statistics of the phase delay, just as a fringe pattern is shifted by a phase retardance in a 1-D interferometer. Thus we 
need to know the spatial structure of the wavefront i.e.: its covariance:

$$
C(\overrightarrow{r \prime})=\langle(A(\vec{r})-\langle A\rangle(A(\vec{r}+\overrightarrow{r \prime})-\langle A\rangle))\rangle .
$$

$A$ is a log-normal process and the turbulence is not strong at astronomical sites. Then one shows (see e.g. (Roddier 1981))that $C$ writes:

$$
C(\vec{r}, \overrightarrow{r \prime})=\left\langle A(\vec{r}) A^{*}(\vec{r}+\overrightarrow{r \prime})\right\rangle=\exp \left(-D_{\phi}(\vec{r}, \overrightarrow{r \prime}) / 2\right)
$$

where $D_{\phi}(\vec{r}, \overrightarrow{r \prime})$ is structure function of the phase.

$$
D(\vec{r}, \overrightarrow{r \prime})=\left\langle(\phi(\vec{r})-\phi(\vec{r}+\overrightarrow{r \prime}))^{2}\right\rangle
$$

$D_{\phi}$ is related to the spatial properties of $n$. D is related to $C$ through:

$$
D(\vec{r}, \overrightarrow{r \prime})=2(C(0)-C(\vec{r}, \overrightarrow{r \prime}))
$$

The structure function of $n$ can be expressed as a function of the scalar distance $r$ :

$$
D_{n}(\vec{r})=C_{n}^{2} r^{\frac{2}{3}}
$$

due to the spectrum of the size of the turbulent eddies, which follows the Kolmogorov law (Kolmogorov 1961) within the so-called inertial range, bounded by the inner scale $l_{0}$ and the outer scale $L_{0}$ of the turbulence. Turbulent eddies cannot develop beyond $L_{0}$, of the order of tens of meters ; it gives a physical meaning to Eq. 7, preventing $D_{n}$ to increase without limit as $r$ increases. On the side of small scales, viscosity dissipates the turbulent eddy energy below $l_{0}$, typically below several millimeters. Since $n$ varies with the altitude above the ground $h$, the refractive index structure constant $C_{n}^{2}$ also varies with altitude.

The phase structure function is given by:

$$
D_{\phi}(\vec{r})=2.91 k^{2} \cos ^{-1}(\zeta)|\vec{r}|^{\frac{5}{3}} \int_{0}^{\infty} C_{n}^{2}(h) d h,
$$

where $\zeta$ stands for the zenith angle of the direction of the source in the sky, and where $k$ is the wave number $k=2 \pi / \lambda$. Fried has introduced the parameter:

$$
r_{0}=\left(\frac{2.91}{6.88} k^{2} \cos ^{-1}(\zeta) \int_{0}^{\infty} C_{n}^{2}(h) d h\right)^{-\frac{3}{5}}
$$


(Fried 1965). Thus Eq.8 can be expressed as a simple function of $r_{0}$ :

$$
D_{\phi}(\vec{r})=6.88\left(\frac{|\vec{r}|}{r_{0}}\right)^{-\frac{3}{5}}
$$

The so-called Fried parameter $r_{0}$ is the key parameter which characterizes the spatial scale of the turbulence, since the spatial properties of the phase are a function of this parameter only. The Fried parameter is the distance across which the root mean square phase of the distorted wave front (with respect to the unperturbed wavefront) is 1 radian. Within this error, the phase is constant and the telescope has the same resolution at $\lambda=500 \mathrm{~nm}$ as a small telescope with a diameter $r_{0}$ in the absence of turbulence. ${ }^{1}$ Note that from Eq. 9, $r_{0}$ varies with the wavelength $\lambda$ :

$$
r_{0} \propto \lambda^{\frac{6}{5}}
$$

An average value of $r_{0}$ at a good astronomical site is $10 \mathrm{~cm}$ at $\lambda=500 \mathrm{~nm}$. The highest values reach $35 \mathrm{~cm}$ some nights at the very best sites.

Now let us come back to our question: what is the angular extension of the image of a point source at the focus of a telescope? From the definition of $r_{0}$, it follows in a straight forward way that the angular image size is $\lambda / r_{0}$.

Then the turbulence limited resolution of a telescope is $\approx 1$ arcsec, or $5 \mu$ radian. In the astronomers jargon, $\lambda / r_{0}$ is the seeing. With a seeing of 1arcsec, the spatial resolution of an 8 meter class telescope is the same as that of a good quality $10 \mathrm{~cm}$ amateur telescope! This frustrating fact justifies the huge efforts going on for more than 30 years to restore the full imaging capabilities of large ground based telescopes.

Fried has defined $r_{0}$ for $\lambda=500 \mathrm{~nm}$. Thus the coherence length at infrared wavelengths is $\approx 60 \mathrm{~cm}$ at $2.2 \mu \mathrm{m}$ and $\approx 160 \mathrm{~cm}$ at $5 \mu \mathrm{m}$, which are the wavelengths of two windows in the absorption spectrum of the atmosphere.

The seeing is weakly dependent on the wavelength (as the one-fifth power of $\lambda$ ). But the degradation factor of the image resolution caused by the turbulence is $\left(\lambda / r_{0}\right) /(\lambda / D)=D / r_{0}$. Typical values of $D / r_{0}$ range from 80 in the visible to 5 at $5 \mu \mathrm{m}$ at an $8 \mathrm{~m}$ telescope. They can be divided by a factor of two during the very best nights at the very best sites. Corrections required to restore diffraction limited images are smaller (and therefore easier to apply) at infrared wavelengths. Indeed, while the optical path, and optical

\footnotetext{
${ }^{1}$ Applying the Maréchal criterion, which considers a variance of the phase of 0.2 radian ${ }^{2}$, the diameter of the non perturbed equivalent telescope is $0.4 r_{0}$.
} 
path fluctuations, are to a first approximation independent of wavelength, there are more waves for a given optical path length at shorter wavelengths. This is why the adaptive optics devices in operation at telescopes in the world are performing mostly at infrared wavelengths.

$r_{0}$ is a critical parameter for adaptive optics. Indeed it drives the spatial sampling of the deformable mirror conjugate to the telescope pupil. Therefore $r_{0}$ also limits the number of photons available per sample to measure the phase delay.

\section{$2.2 \quad$ Temporal properties}

Temperature inhomogeneities in the atmosphere are not stationary in space. Turbulent layers are swept along by the wind. To first approximation, one can consider that the temperature distribution pattern is simply translated : this is the Taylor hypothesis (Taylor 1938). The instantaneous phase screen translates across the pupil. We can define a coherence time of the wavefront, which is the time a coherent area crosses a point of the pupil:

$$
\tau_{0, \text { wavefront }}=r_{0} / v
$$

where $v$ is the wind velocity at the altitude of a turbulent layer. Often, there are a few layers. In this case:

$$
\tau_{0, \text { wavefront }}=r_{0} / \Delta v
$$

where $\Delta v$ is the vertical dispersion of the wind velocity. Values of $\tau_{0, \text { wavefront }}$ are of the order of $10 \mathrm{~ms}$ at visible wavelengths. Since $r_{0}$ varies with $\lambda 60 \lesssim$ $\tau_{0, \text { wavefront }} \lesssim 160 \mathrm{~ms}$ in the infrared range $2.2 \lesssim \lambda \lesssim 5 \mu \mathrm{m}$. Significantly longer coherence times are observed at the best astronomical sites.

The coherence time depends on the scale of the wavefront perturbation. The largest scale is the slope of the wavefront across the whole pupil (the so-called tilt); it is proportional to the gradient of $n$. The tilt coherence time is:

$$
\tau_{0, t i l t}=12.33\left(\frac{D}{r_{0}}\right)^{1 / 6}\left(\frac{r_{0}}{v}\right)
$$

(Tyler 1994; Schöck et al. 2002). At an 8m telescope in a good astronomical site, $r_{0} \approx 0.15 \mathrm{~m}$ and $v \approx 30 \mathrm{~m} / \mathrm{s}, \tau_{0, \text { tilt }}$ ranges from $0.12 \mathrm{~s}$ at $\lambda=0.5 \mu \mathrm{m}$ to $1.2 \mathrm{~s}$ 
at $\lambda=5 \mu \mathrm{m}$. It scales as $\lambda$. Thus $\tau_{0, t i l t}$ is approximately 10 times larger than the wavefront coherence time.

Both these coherence times are crucial parameters for adaptive optics devices, since they limit the maximum integration time for collecting photons to measure the wavefront surface.

\subsection{Flux requisite for adaptive optics}

The basic question an astrophysicist has to answer to evaluate the usefulness of adaptive optics for astrophysical imaging is : Is the probability high enough to get a given Strehl ratio, or a given FWHM, for her/his programme sources. One needs to know the number of photons required for the wavefront sensor. Several sources of errors or bias contribute to the error budget of the incoming wavefront measurements (Parenti \& Sasiela 1994; Le Louarn et al. 1998). We now briefly review these errors.

Adaptive optics devices currently in use in astrophysical observatories consist of two adaptive components that control the wavefront: the tip-tilt mirror, which corrects for the global slope of the wavefront across the whole telescope pupil, and the deformable mirror, which corrects the higher orders of the corrugated wavefront. The wavefront error budget is split between the tilt and higher-order wavefront corrector. Ideally, the only unavoidable noise is photon noise. The expression for the variance of tilt errors is:

$$
\sigma_{p h_{t}}^{2}=\frac{8 \pi h c}{3 \lambda_{t} \rho_{t} \tau_{d, t} D^{2} I_{t}}\left(\frac{\lambda_{s c i}}{\lambda_{t}}\right)^{\frac{12}{5}}
$$

where $\lambda_{t}$ and $\lambda_{s c i}$ are the wavelengths respectively of the tilt sensor and of the science channel, $\rho_{t}$ is the efficiency of the tilt channel (optics and detector), $\tau_{d, t}$ is the delay of the tilt correction, and $I_{t}$ is the irradiance at the tilt sensor.

And for the modes higher than tilt, the phase error variance is:

$$
\sigma_{p h_{h o}}^{2}=\frac{4 \pi^{2}}{3}{\frac{k_{s c}}{k_{w f s}}}^{2} \frac{h c k_{w f s}}{2 \pi \rho_{w f s} \tau_{d} d_{s}^{2} I_{w f s}}
$$

where $\rho_{w f s}$ is the efficiency of the wavefront sensor channel, $\tau_{d}$ is the integration time of the wavefront sensor, $d_{s}$ is the size of a square subpupil of the (Shack-Hartmann) wavefront sensor, and $I_{w f s}$ is the irradiance of the wavefront sensor.

Unfortunately there are several other sources of error. They are 
- additive noise, such as detector readout noise and sky background;

- delay time error, due to the detector integration time and signal processing time which decreases the close loop bandwidth;

- fitting error, due to the finite spacing of actuators of the deformable mirror which prevent correction of aberrations at spatial frequencies higher than $\left(2 d_{s}\right)^{-1}$;

- aliasing error, also due to the finite spacing of actuators of the deformable mirror. It results in turning back high order parts of the phase spectrum in the low order range.

The long exposure Strehl ratio $S_{l e}$ resulting from these wavefront errors writes:

$$
S_{l e}=\frac{\exp \left(\sigma_{h o}^{2}\right)}{1+\pi^{2} / 2\left(D / \lambda_{s c}\right)^{2} \sigma_{\text {tilt }}^{2}}+\frac{1-\exp \left(-\sigma_{h o}^{2}\right)}{1+\left(D / r_{0}\right)^{2}}
$$

The first term of Eq. 17 is the Strehl of the close to diffraction limited component of the image, also called its coherent core. The second one describes the Strehl of the uncorrected component, also called the incoherent halo.

In the $\mathrm{K}$ photometric band $(\lambda=2.2 \mu \mathrm{m}, \Delta \lambda=0.4 \mu \mathrm{m})$, with $r_{0}=0.2 \mathrm{~m}$, and $89 \%$ of the seeing being produced at the comparatively low altitude $h$ $=2.5 \mathrm{~km}$, the Strehl at one of the VLT $8 \mathrm{~m}$ telescope is expected to be $S_{l e} \approx 0.86$ at most, not 1 because of aliasing and fitting errors, and additive (mostly readout) noise. This value is obtained as long as photon noise dominates other errors, i.e. up to magnitude in the $\mathrm{R}$ photometric band $(\lambda=0.7 \mu \mathrm{m}, \Delta \lambda=0.22 \mu \mathrm{m})$ of $m_{R} \approx 10$, i.e. as long as the wavefront sensor collects:

$$
N_{p h} \approx 134 \text { photons. } \mathrm{s}^{-1} \cdot \mathrm{cm}^{-2} .
$$

At lower fluxes, $S_{l e}$ decreases rather steeply.

\subsection{Isoplanatic patch}

When the programme object is not bright enough to feed the wavefront sensor of an adaptive optics device, one has to use another reference source. It cannot be far from the programme object, because the wavefronts from 

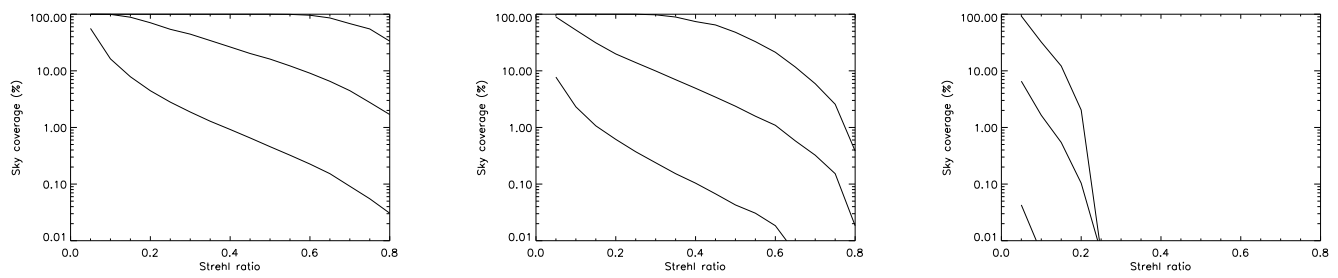

Figure 2: Sky coverage of an adaptive optical system at an $8 \mathrm{~m}$ telescope. Pupil sampling by the actuators of the deformable mirror : $0.5 \mathrm{~m} . r_{0} \approx 0.17 \mathrm{~m}$. From left to right: in the $\mathrm{K}$ photometric band $(2.2 \mu \mathrm{m})$, in the $\mathrm{J}$ band $(1.25 \mu \mathrm{m})$ and in the $\mathrm{V}$ band $(0.55 \mu \mathrm{m})$. From top to bottom: in a direction of galactic latitude $b=0^{\circ}$ (the Milky Way), $b=20^{\circ}$ and $b=90^{\circ}$ (the galactic pole). Courtesy of M. Le Louarn.

the programme object and from the reference source must have undergone similar perturbations. The isoplanatic angle $\theta_{0}$ defines the angle between the directions of two incoming wavefronts for which the correlation is half the autocorrelation of one of them. $\theta_{0}$ can be derived from $r_{0}$. In case of a single thin turbulent layer at altitude $h$ it is $\theta_{0}=r_{0} / h, \approx 2$ arcsec. A more realistic expression has to take into account the vertical distribution of the turbulent layers:

$$
\theta_{0}=r_{0} / f(h)
$$

where $f(h)$ is the normalized $5 / 3$ momentum of the $C_{N}^{2}$ vertical distribution:

$$
f(h)=\left(\frac{\int_{0}^{\infty} C_{n}^{2}(h) h^{5 / 3} d h}{\int_{0}^{\infty} C_{n}^{2}(h) d h}\right)^{3 / 5}
$$

(Fried 1979; Roddier 1981). $\theta_{0}$ scales as $\lambda^{6 / 5}$. Typical values of $\theta_{0}$ are 2 -4arcsec in the visible and $\approx 30$ arcsec in the infrared.

\subsection{Sky coverage}

From the density function of the stars in the sky and the isoplanatic patch, one computes the probability to find in a given direction a star which provides the flux required per coherent area and per coherence time for a given Strehl ratio.

Figure 2 shows some examples computed with instrument and seeing parameters typical of the Very Large Telescope built by the European Southern 
Observatory in Northern Chile. In the infrared K photometric band $(2.2 \mu \mathrm{m})$ the sky coverage is statistically complete in the directions of the Milky Way (galactic latitude $b=0^{\circ}$ ); it is less than $1 \%$ at moderate Strehl ratio towards the galactic pole. At $1.25 \mu \mathrm{m}$ ( $\mathrm{J}$ band), the coverage is similar. But in the visible the sky coverage is unacceptably low whatever the direction.

This poor situation led astrophysicists to imagine to create an artificial star where they need it (Foy \& Labeyrie 1985). This is the laser guide star (hereinafter referred as LGS). Before it is published in the open literature, this concept started to be developed in American military laboratories within the framework of the Strategic Defense Initiative programme; there, it was classified until 1991.

The principle of the LGS is simple. A laser beam is directed towards the programme source. The beam is backscattered in the atmosphere, producing a spot in the sky as viewed from the telescope. This spot is used to feed the wavefront sensor of the telescope adaptive optics device. In the following we will review the possible backscattering processes.

\section{Backscattering processes in the Earth at- mosphere}

The three physical processes which can be envisioned to backscatter laser light in the atmosphere are shown in Fig.3:

\subsection{Mie scattering}

Mie scattering is caused by small particles, with a size large with respect to $\lambda$. They are dust, including sometimes volcanic ashes in the stratosphere (Fugate et al. 1991), aerosols and moisture. LIDAR techniques to analyze the microconstituants of the lowermost atmosphere rely on Mie scattering. Most of these scatterer are located under the inversion layer, typically at $\approx 2000-4000 \mathrm{~m}$. Therefore Mie scattering is generally not relevant for a LGS, since it would not allow to sample the turbulent layers above this relatively low altitude, which significantly contributes to the seeing (see Fig.1). 


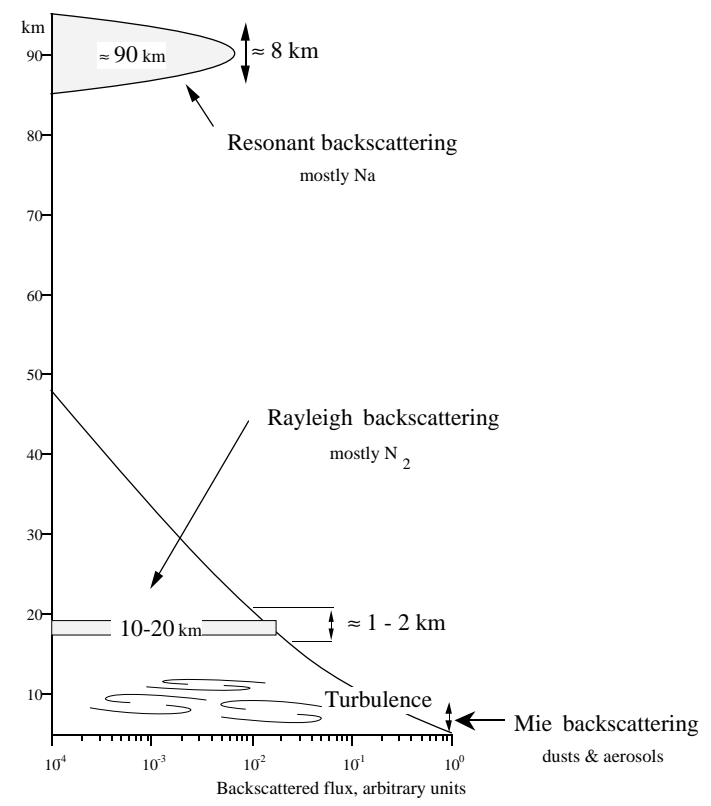

Figure 3: Backscattering processes in the atmosphere. Return photons versus altitude are given, in arbitrary units. Courtesy of M. Tallon

\subsection{Rayleigh scattering}

Air molecules scatter light. It is mostly nitrogen $N_{2}$ since it is the most abundant molecule in the atmosphere. Pulsed lasers have to be used to avoid confusion between return photons from high layers with those returned from low altitude ones. Usually, time gates allows us to select a 1 to $2 \mathrm{~km}$ thick layer $\approx 20-30 \mathrm{~km}$ high, depending on the excitation wavelength $\lambda_{e}$. Rayleigh scattering efficiency varies as $\lambda_{e}^{-4}$. It favors short wavelength lasers. The return flux from altitude $h$ is the product of the Rayleigh backscattering cross section by the molecule density at this altitude $h$ :

$$
\sigma_{B}^{R} n_{R}(h)=3.610^{-31} \lambda_{e}^{-4.0117} P(h) / T(h) \mathrm{m} \cdot{ }^{-1} \mathrm{sr}^{-1}
$$

where $\mathrm{P}$ is the atmospheric pressure in mbar, $\mathrm{T}$ is the temperature in $\mathrm{K}$ (Gardner et al. 1986). Rayleigh LGSs have been experimented with a dye laser at the Lincoln Lab (Primmerman et al. 1991), with a copper vapor laser at the Starfire Optical Range (Fugate et al. 1994), with a doubled frequency NdYAG laser at Observatoire de la Côte d'Azur(France)(Séchaud et al. 1988), and with an ultraviolet excimer laser at Mount Wilson Observatory (California) (Thompson \& Xiong 1995). It is not the most popular backscattering process because of relatively low altitude of the laser spot (see below Section 5. 


\subsection{Resonant scattering}

Fluorescence can be excited with a laser beam locked to atomic excitation lines. Several transitions of different atoms can be involved. The optimal choice has to take into both the atom cross section of the transition and the abundance of the specie. Table 3.3 compares sodium, potassium and iron resonance lines and column densities (Papen, Gardner, \& Yu 1996).

Table 1: Cross section of resonant lines $\sigma$ and column density of sodium, potassium and iron free atoms.

\begin{tabular}{|c||c|c|c|}
\hline & $\mathrm{Na}$ & $\mathrm{K}$ & $\mathrm{Fe}$ \\
\hline \hline$\lambda(\mathrm{nm})$ & 589 & 769 & 372 \\
$\sigma\left(\mathrm{cm}^{2}\right)$ & $1.110^{-9}$ & $1.010^{-9}$ & $1.010^{-10}$ \\
$N\left(\mathrm{~cm}^{2}\right)$ & $510^{9}$ & $310^{8}$ & $110^{10}$ \\
\hline
\end{tabular}

The potassium resonance line is embedded in telluric $\mathrm{O}_{2}$ absorption lines. Table3.3 shows that the most suitable resonant process is fluorescence of the sodium line $D_{2}$ at $589 \mathrm{~nm}$. When the single valence electron of the sodium atom in the mesosphere absorbs a laser photon at $589 \mathrm{~nm}$, it jumps from the $2 S_{1 / 2}$ ground energy level to the $3 P_{3 / 2}$ first level. Due to spontaneous emission, the valence electron returns to the ground level emitting a photon at the same wavelength.

Whether this process provides enough return photons to feed the wavefront sensor of an adaptive optics depends on both the properties of the $D_{2}$ transition and the physical parameters of the mesosphere.

\subsection{Properties of the sodium layer in the mesosphere}

The so-called sodium layer in the mesosphere is located from 75 to $110 \mathrm{~km}$ above sea level. Sodium atoms may be produced by dissociation of $\mathrm{NaCl}$ molecules, which come from the innermost atmospheric layers ; but it is rather difficult to account for their move to the mesosphere through the purely radiative stratosphere. A more popular explanation of mesospheric sodium atoms is ablation of meteorites. When meteorites enter Earth's atmosphere, their average temperature at $\approx 100 \mathrm{~km}$ allows sodium atoms to be freed (Slipher 1929; Clemesha et al. 1978; Jegou et al. 1985a; Jegou et al. 
1985b).

The average altitude $H$ of the sodium layer varies with season (Bowman, Gibson, \& Sandford 1969; Megie \& Blamont 1977). In winter, it is $\approx 89 \mathrm{~km}$, and the temperature is $210 \pm 25 \mathrm{~K}$; in summer, it is $\approx 91 \mathrm{~km}$ and the temperature is $160 \pm 25 \mathrm{~K}$. The sodium column density $\rho_{N a}$ varies from 2 to $1010^{9}$ atoms.cm ${ }^{-2}$ from summer to winter (Blamont \& Donahue 1961; Gardner et al. 1986). For comparison the density of molecules at $90 \mathrm{~km}$ is $\approx 710^{13} \mathrm{~cm}^{-3}$. Therefore the total mass of free sodium in the layer amounts to $\approx 1$ metric ton; this is quite small, so that one could think about feeding it using rockets, but ... Note also that there are approximately 10 times more sodium atoms bound within aggregates (Wuerker 1997)

Sporadic events in the sodium distribution have been frequently reported. Within thin layers ( $\approx 1 \mathrm{~km}$ thick or less), the local column density abruptly increased for minutes (von Zahn \& Hansen 1988; O'Sullivan et al. 2000; Michaille et al. 2000). Sporadics could be due to convective cells entering the sodium layer. It slightly affects the weighted average altitude of the sodium layer.

The influx of sodium atoms is $\approx 1.610^{4} \mathrm{~cm}^{-2} \cdot \mathrm{s}^{-1}$. The mean residence time of sodium in the layer is $\approx 6$ days: ... such a short time precludes feeding the sodium layer with rockets. Two loss mechanisms have been proposed:

- $\mathrm{Na}$ transforms to $\mathrm{NaO}_{2}$ through the reaction

$$
\mathrm{Na}+\mathrm{O}_{2}+\mathrm{M} \rightarrow \mathrm{NaO}_{2}+\mathrm{M},
$$

where M stands for a neutral atom (Swider 1985). The mass loss rate is proportional to the squared metal atom density, and is an inverse function of temperature.

- $\mathrm{Na}$ contributes to the formation of cluster ions $\mathrm{Na}^{+} .\left(\mathrm{H}_{2} \mathrm{O}\right)_{n}$ (Richter \& Sechrist 1979b; Richter \& Sechrist 1979a; Jegou et al. 1985a). The mass loss rate is again an inverse function of temperature, and also a function of zonal winds.

Both mechanisms account for the observed seasonal variations of the sodium column density because of the correlated temperature variations. Molecules or particles which are formed settle slowly gravitationally and leave the sodium layer. 
The time between collisions of a sodium atom, mostly with nitrogen molecules, is $\approx 100 \mu \mathrm{s}$. Mean time between collisions with $O_{2}$, the dominant paramagnetic species, is $140 \mu \mathrm{s}$

The reader interested in more information about the properties of the sodium layer should refer e.g. to the paper (Happer et al. 1994).

\subsection{Properties of the sodium $D_{2}$ transition}

The absorption to the $3 P$ level is an electric dipole transition. This scattering process slightly favors the emission towards both directions along the direction of propagation of the exciting field ; the resulting slight backscattering enhancement is neglected.

Figure 4 shows the detailed structure of the energy levels $3 S_{1 / 2}$ and $3 P_{3 / 2}$. Both levels are characterized by the principal quantum number $n=3$ of the valence electron. For the ground state, the electron orbital angular momentum is $l=0$, so that, given the electron spin angular momentum quantum number $S=1 / 2$, the total electron angular momentum quantum number is $J=1 / 2$. The nuclear spin angular momentum quantum number is $I=3 / 2$. Therefore the total angular momentum quantum number of the ground state has two values $F=I \pm J=1$ or 2 . These two sublevels have different energies because of the magnetic interaction between the electron and the nuclear dipoles; this results in the hyperfine structure of the $D$ line (Fig 4), of which the two components are $1.772 \mathrm{GHz}$ apart.

The first excited level has an electron orbital angular momentum quantum number 1 ; hence the total electron angular quantum number $J=S \pm l$ has two values: $J=1 / 2$ and $J=3 / 2$, which gives the two sublevels $3 P_{1 / 2}$ and $3 P_{3 / 2}$. Their energies differ by $\Delta E=520 \mathrm{GHz}$, because of the spin orbital interaction which is the magnetic interaction between the electronic spin and the orbital momenta. This causes the splitting of the $D$ line into the $D_{1}$ and the $D_{2}$ lines respectively. The ratio of the probabilities of the two hyperfine transitions is $3 / 5$. Therefore because of this lower probability, until now backscattering from the $D_{1}$ line at $589.6 \mathrm{~nm}$ has never been considered for the LGS.

The interaction between the total electron magnetic momentum and the nuclear magnetic momentum makes a further splitting of the $3 P$ levels. The $3 P_{1 / 2}$ level splits into $F=1$ and $F=2 ; 3 P_{3 / 2}$ splits into $F=0,1,2$, and 3. The difference of energy between $F=0$ and $F=3$ is $0.112 \mathrm{GHz}$, which is much less than its counterpart for the ground level. This property will be 

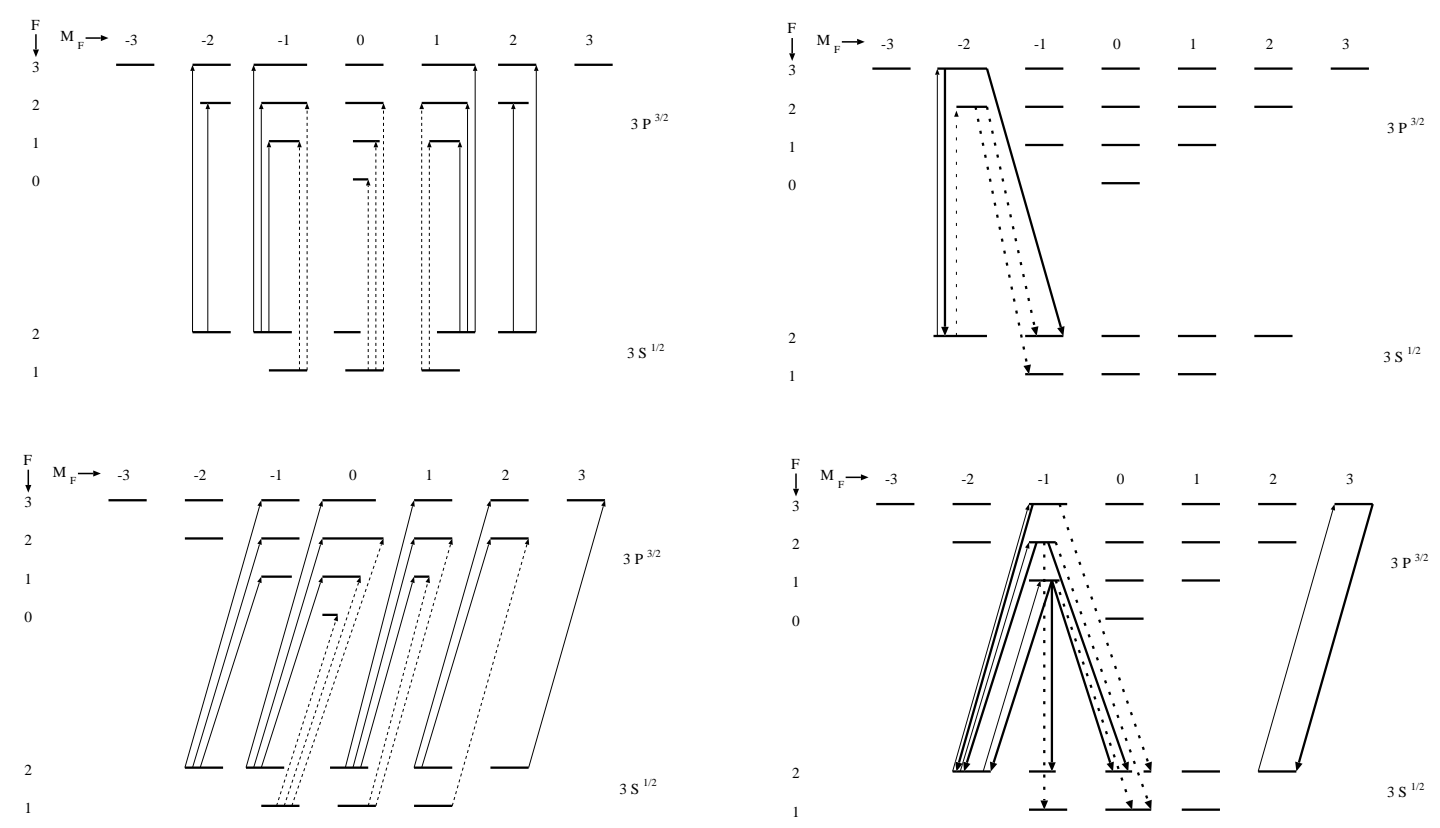

Figure 4: Energy level diagram of the single valence electron of the sodium atom for the $D_{2}$ transition.

Top: linearly polarized excitation $\left(\Delta M_{F}=0\right)$; left: all allowed transitions; right, example $\Delta M_{F}=0,-1$ and +1 radiative decays showing how the population of the level $3 S_{1 / 2}\left(F=1 ; M_{F}=0\right)$ is enhanced with respect to the other levels; transitions from this level have the lowest probabilities; therefore after a few excitation-desexcitation cycles, the absorption in the mesosphere decreases.In addition, if one excites the $3 P_{3 / 2}$ level only from the sublevel $F=2$ of the ground state, with a bandwidth $\lesssim 1 \mathrm{GHz}$, then those atoms which decay down to the $2 S_{1 / 2} F=1$ sublevel cannot absorb again, resulting in a depopulation of $F=2$ sublevel, and therefore in a decrease of the return flux.

Bottom: left circularly polarized excitation; left: all the allowed transitions; right showing in this case the population of the $3 S_{1 / 2}\left(F=2 ; M_{F}=2\right)$ is enhanced; the $D_{2}$ transition from this level has the highest probability; therefore after a few cycles, the $D_{2}$ absorption increases. 
discussed below to explain the optical pumping of the ground sublevels.

Zeeman splitting of each $\mathrm{F}$ level into the $2 F+1$ integer values in the interval $(-F ; F)$ occurs when the atom is in presence of a magnetic field. They are the $M_{F}$ Zeeman sublevels. The average Earth magnetic field is $\vec{B} \approx 0.5 \mathrm{G}$, and the corresponding average Zeeman splitting is $\approx 0.35 \mathrm{MHz}$.

Selection rules easily derived from the requirement that the system made of an absorbing atom and an absorbed photon conserves its total (i.e.: atom + photon) energy and angular momentum during the absorption. A photon carries its well-known energy $h \nu$ and the unit angular momentum $h$. Of course, the atom's angular momentum after the absorption of the photon has to correspond to an existing sublevel. Thus available transitions have to satisfy $\Delta F=-1,0$ or +1 . It also applies to the Zeeman sublevel. The population of the sublevels is sensitive to the polarization of the exciting electric field. If the laser beam is linearly polarized, the quantization axis is parallel to the electric field vector of the beam, and thus allowed transitions satisfy the condition $\Delta m_{F}=0$; if it is circularly polarized, the quantization axis is parallel to the beam propagation direction and thus $\Delta m_{F}= \pm 1$ for left and right polarization. For left (right) polarized light, this absorption pattern in Fig. 4 works if the direction of the beam propagation is (anti) parallel to the Earth magnetic field $\vec{B}$.

The lifetime of the $3 P_{3 / 2}$ level is $\tau=16 \mathrm{~ns}$, and the corresponding homogeneous linewidth of the $D_{2}$ transition is given by

$$
\Delta \nu_{H}=2 /(4 \pi \tau)=10 \mathrm{MHz}
$$

This is much smaller than the Doppler linewidth:

$$
\Delta \nu_{D}=2 \nu_{0} / c \sqrt{\ln 2} \sqrt{2 R T / A}
$$

which ranges from 0.96 to $1.1 \mathrm{GHz}$ depending on the temperature $\mathrm{T}$ which varies with the season. As usual, $R$ is the gas constant, $A=23$ the atomic mass and $\nu_{0}$ the central frequency of the line. Figure 5 shows the profile of the hyperfine structure of the $D_{2}$ mesospheric line.

The peak cross section of the $D_{2}$ transition for homogeneous linewidth is $\sigma_{h}=1.1410^{-9} \mathrm{~cm}^{2}$. Therefore it is $\sigma_{D}=1.1410^{-9} \times \Delta \nu_{H} / \Delta \nu_{D} \approx 10^{-11} \mathrm{~cm}^{2}$ for the Doppler width.

Finally, it is useful for what follows to mention power broadening of the energy levels due to the laser electric field. This broadening is expressed as 


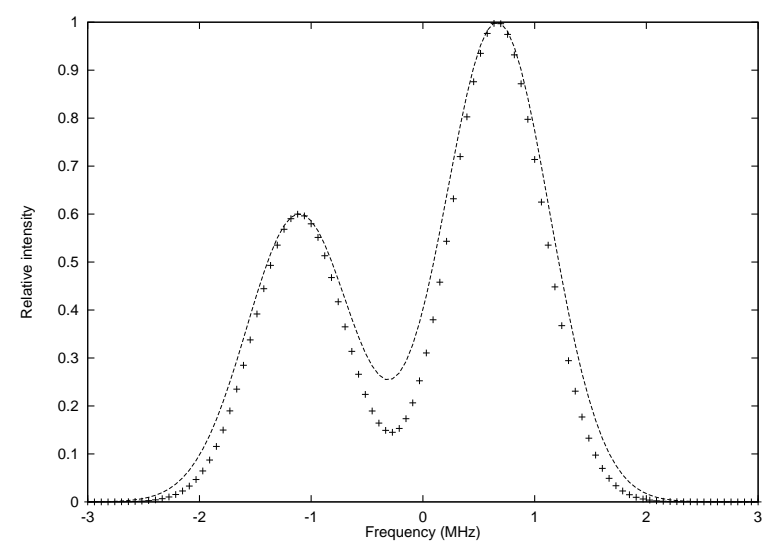

Figure 5: The profile of the mesospheric $D_{2}$ line showing the splitting due to the hyperfine structure of the ground state. +'s line: Winter time; dotted line: Summer time.

the Rabi frequency $\Omega$, which is proportional to the transition dipole momentum and to the square root of the number of photons:

$$
\Omega=\sqrt{P}
$$

Optical pumping There are more transitions toward $3 P_{3 / 2}(F=0,1,2,3)$ at $\Delta M_{F}=0$ from $3 S_{1 / 2}(F=2)$ than from $3 S_{1 / 2}(F=1)$. There are 3 and 5 Zeeman sublevels in the levels $F=1$ and $F=2$ respectively, and from 1 to 7 for the $3 P_{3 / 2}(F=0,1,2,3)$ sublevels. Therefore several sublevels cannot be populated with a $\Delta F=0$ excitation, e.g. $M_{F}= \pm 3$ from $3 S_{1 / 2}(F=2)$ or $F=3, \forall M_{F}$ from $3 S_{1 / 2}(F=1)$. Excited atoms decay following the classical selection rules: $\Delta M_{F}=0$ or \pm 1 and $\Delta F=0$, except for both $\Delta M_{F}=0$ and $\Delta F=0$ simultaneously. After a few excitation-decay cycles, there is a trend to depopulate the outermost $M_{F}$ sublevels, to the $3 S_{1 / 2}(F=1)$ sublevel's benefit. Since the transition probabilities are lower from $3 S_{1 / 2}(F=1)$ than from $3 S_{1 / 2}(F=2)$, the absorption in the sodium layer decreases; this holds as long as some process (see below) resets the sublevels population distribution.

If the laser spectral bandwidth is $1 \mathrm{GHz}$ or less, locked on the central wavelength of $3 S_{1 / 2}(F=2) \Longrightarrow 3 P_{3 / 2}$. Part of the excited atoms decay to the $3 S_{1 / 2}(F=1)$ ground state, from which they cannot be excited again. After a few excitation cycle the $F=2$ ground level is depopulated. Even 
the excitation to the $3 P_{3 / 2(F=3)}$, from which the only possible decay is to the $(F=2)$ ground state, suffers from the competition with the off-resonant excitation of $3 P_{3 / 2(F=2)}$ which can decay to $F=1$ ground state, which is $59.8 \mathrm{MHz}$ apart, not far enough compared to $\Delta \nu_{H}$. Thus no absorption is possible until the populations are rethermalized. Therefore linearly polarized laser beams are not that efficient.

Population inversion holds as long as one of the following redistribution processes destroy it:

- winds in the mesosphere, with a velocity of $\approx 30 \mathrm{~m} / \mathrm{s}$, renew the volume of sodium atoms typically every 0.016 s for a $1^{\prime \prime}$ seeing,

- the diurnal motion of the astronomical source, which forces the laser beam to scan the mesosphere to track it. The scan velocity is a function of the latitude $L$ of the observatory, and of the coordinates of the source; it is $\approx 460 \cos L \mathrm{~m} / \mathrm{s}$ for a source at zenith observed from a site at latitude $L$. Thus the sodium volume is renewed every $\approx 1 \mathrm{~ms}$ with an $1^{\prime \prime}$ seeing.

- spin exchange between the valence electron and paramagnetic molecules, mostly $\mathrm{O}_{2}$ (Happer et al. 1994). Relaxation time would be $\approx 0.01 \mathrm{~s}$ (Kibblewhite 2000).

On the other hand, with circularly polarized light, transitions $\Delta M_{F}= \pm 1$ are involved. Only circularly polarized beams can reach the $3 P_{3 / 2\left(F=3, M_{F}=3\right)}$ Zeeman level. It decays to $3 S_{1 / 2}\left(F=2, M_{F}=2\right)$ ground sublevel: this transition has the highest probability. This optical pumping can go on indefinitely.

Thus the absorption from both $3 S_{1 / 2}(F=1)$ and $(F=2)$ is more efficient than with linearly polarized light, and an indirect optical pumping from $F=1$ to $F=2$ occurs. Therefore the emitting laser should be circularly polarized, leading to a gain in the return flux of $\approx 50 \%$. Measurements on the sky show an increase o $\approx 30 \%$ when a circular polarization is used (Jeys et al. 1992).

\subsection{Saturation of $D_{2}$ absorption}

An atom cannot absorb more than one photon per cross section unit and per lifetime of the transition. Thus the saturation intensity $I_{s}$ and the flux of absorbed photons at the mesosphere is: 


$$
I_{s}=h \nu / \sigma_{h} \tau=185 \mathrm{~W} / \mathrm{m}^{2} \text { or } \Phi_{s}=1 / \sigma_{h} \tau=5.510^{16} \text { photons } / \mathrm{s} / \mathrm{cm}^{2}
$$

where $\sigma_{h}$ is the peak cross section for a single frequency (homogeneous width) beam. Integrating over all the velocity groups spanning the Doppler profile of the $D_{2}$ transition leads to the absorption at saturation:

$$
A=\frac{\rho_{h} / \tau}{\Phi_{s}} \frac{\Delta \nu_{h}}{\Delta \nu_{t}} \approx 2 \%
$$

where $\Delta \nu_{t}=3 \mathrm{GHz}$ stands for the total width of the $D_{2}$ line, including the ground state hyperfine splitting.

Thus, below saturation absorption increases as the number of photons available, i.e. proportionally to the laser power. Beyond the saturation of the absorption for a homogeneous bandwidth laser beam, absorption is still increasing. Indeed power broadening allows atoms of which the velocities are off the laser spectral bandwidth to absorb. Since the Rabi frequency varies as the square root of the power (Eq. 24), absorption does also. It holds as long as the Rabi frequency is less than the Doppler broadening for a single mode laser. If the laser spectral profile is modulated, then absorption is no longer significantly increasing as the Rabi frequency reaches the distance between modes. Further increase of the absorption is due to the absorption in the wings of the Doppler profile, which are in theory indefinitely extended.

\section{The tilt determination}

The tilt is the phase gradient across the entire telescope pupil. It results in image wandering at the telescope focus. For the case of a laser beam emitted through the telescope itself, the beam undergoes tilt deflection on upward propagation the same as does the backscattered light. The round trip time to the mesosphere is $\approx 0.6 \mathrm{~ms}$ at zenith. It is $\approx$ two orders of magnitude shorter than the tilt coherence time defined in Eq. 14. Thus both deflection to and from the mesosphere are approximately equal. As a result the apparent location of the LGS is fixed with respect to the telescope optical axis ; one does not know what is the location of the LGS in the sky and the tilt cannot be measured. In the case the beam is propagated from an auxiliary telescope apart from the main one, the apparent location of the LGS is the sum of the 
tilts in front of both telescopes. Both are unknown. Thus in this case also the tilt cannot be measured.

The rms value of the tilt in units of arcsec is:

$$
\sigma_{\theta}=0.062 D^{-1 / 6} r_{0}^{-5 / 6} \cos z^{-2}
$$

where $D$ stands for the telescope pupil diameter and $r_{0}$ is the Fried parameter, both units of meters, and $z$ is the zenith distance(Roddier 1981). The $D^{-1 / 6}$ term is due to the variation of the phase gradient with the baseline according to the structure function of the air refraction index (see Eq. 7). At an 8-m telescope, $\sigma_{\theta}=0.21$ and $0.43^{\prime \prime}$ respectively at zenith and at $45^{\circ}$ zenith distance for $r_{0}=0.15 \mathrm{~m}$. These values are much larger than the telescope diffraction limit, which is 0.055 and 0.014 ", respectively, in the $\mathrm{K}$ band $(2.2 \mu \mathrm{m})$ and in the $\mathrm{V}$ band $(550 \mathrm{~nm})$. Tilt carries $\approx 87 \%$ of the variance of the departure of the corrugated wavefront from a plane (Rigaut \& Gendron 1992). Definitely, it must be measured and corrected for an adaptive optics device to deliver a diffraction limited image. Indeed, let us assume the incoming corrugated wavefront is corrected for orders higher than the tip-tilt with a LGS-fed adaptive optics device. Diffraction limited images move on the detector as soon as the exposure time is larger than the coherence time of the wavefront for the tilt. Thus integrating on the detector (typically several minutes or $\approx$ one hour)would hugely degrade the spatial resolution: one would pay for an adaptive optics device and for a LGS for a marginal gain in the resolution.

Several solutions have been proposed. We will review in the following those which are being developed or implemented. They are i/ the use of close by natural guide star, ii/ the polychromatic LGS and iii/ the combined Rayleigh and sodium LGS.

\subsection{The tilt from a natural guide star}

When the tilt indetermination had been identified(Pilkington 1987; Séchaud et al. 1988), it has been proposed to use a natural guide star (NGS). It was expected that the NGS could be much fainter and farther apart from the programme object than required for high-order mode measurements; indeed for the tip and tilt Zernike modes, both the entire pupil can be used and the tilt isoplanatic patch is larger than that of higher orders. Thus the probability to find such a NGS was thought to be reasonably high. Unfortunately this 


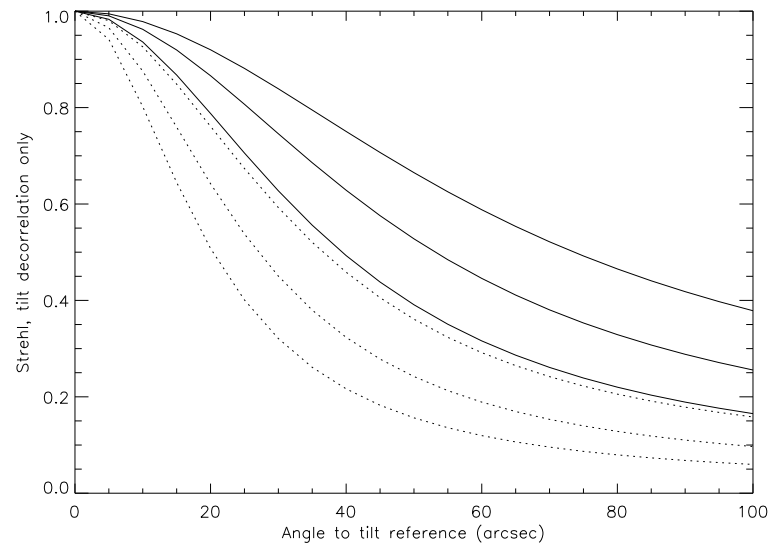

Figure 6: Attenuation of the Strehl ratio versus the distance to the reference object for the tilt. Solid lines: good seeing model at Cerro Paranal. Dotted lines: medium seeing. For each set of curves, top: $2.2 \mu \mathrm{m}$, medium: $1.65 \mu \mathrm{m}$ and bottom: $1.25 \mu \mathrm{m}$. (Le Louarn et al. 1998)

is false as pointed out by Rigaut and Gendron (Rigaut \& Gendron 1992), because of the huge contribution of the tilt to the phase variance.

Therefore the required flux for the NGS is such that the contribution of the photon noise is negligible with respect to the phase error. It severely constrains the limiting magnitude of the tilt NGS. In the same way, the relative anisoplanatism error must be significantly smaller than for the higher modes which precludes using NGS's rather far apart. Figure 6 shows the attenuation of the Strehl ratio as a function of the distance of the tilt reference to the programme object.

Figures 7 and 8 compare the Strehl ratio versus the object magnitude for an adaptive optics device fed with a NGS solely or with a LGS for orders higher than the tilt and with an NGS for the tilt.

For bright objects, the Strehl ratio is lower with a LGS because of the cone effect (see Section 5. Then, as soon as the photon noise affects the NGS-fed device, the LGS-fed device leads to better performances for quite a small magnitude interval: the drop in the Strehl ratio with the LGS-fed device is due to the tilt anisoplanatism.

This method is the only one ready to be implemented on a telescope. It was in use at Calar Alto (Davies et al. 2000), and it is at Lick (Olivier et al. 1999), and now at the Keck (Wizinowich et al. 1998). It is planed on the relatively short term for the other large telescope projects. Its drawback is the low sky coverage it provides as shown in Fig. 9. If the sky coverage is 


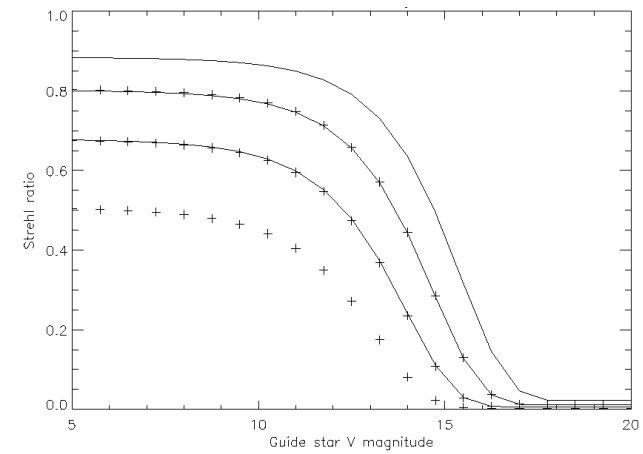

Figure 7: Strehl ratio versus magnitude at $\lambda=0.55 \mu \mathrm{m}$ ) of the reference star for the adaptive optics device. There is no laser guide star. Solid lines: good seeing conditions at Cerro Paranal. Crosses: medium seeing. Set of curves as in Fig. 6 . (Le Louarn et al. 1998).

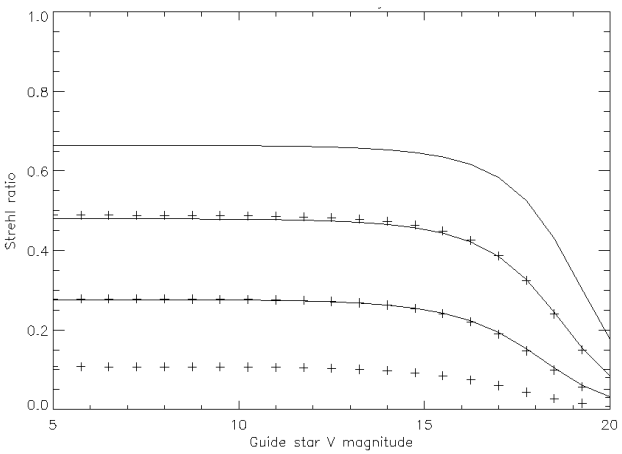

Figure 8: Same as Fig. 7 with a laser guide star for correction of the Zernike polynomials higher than the tilt order and a NGS for the tilt.

good at $2.2 \mu \mathrm{m}$ even in the galactic pole direction $\left(b=90^{\circ}\right)$, it is totally insufficient in the visible, even in the most favorable case of the Milky Way directions $\left(b=0^{\circ}\right)$.

\subsection{The Polychromatic Laser Guide Star}

\subsubsection{The principle}

The polychromatic laser guide star (PLGS) relies on the chromatic properties of the refraction index of the air $n$ (Fig. 10) to overcome the tilt problem. In particular, in the ultraviolet this variation is rather abrupt. Thus a LGS emitting in the mesosphere at several wavelengths, including at least one in the ultraviolet will appear as located at several slightly different locations at the focus of the receiving telescope, because the differences in the optical paths due to the variation in $n$. Thus one can observe a differential tilt whereas the tilt itself remains not observable (see Fig. 11).

The tilt, $\theta$, can be derived from the differential tilt, $\delta \theta$, thanks to the principle of separability of variables applied to $n$ (Filippenko 1982). It allows 

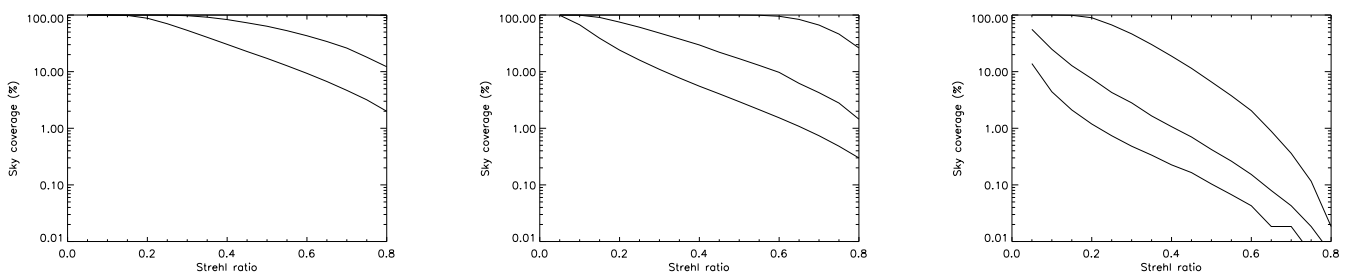

Figure 9: Sky coverage as a function of the Strehl ratio with a monochromatic LGS at $2.2 \mu \mathrm{m}$ (left), $1.25 \mu \mathrm{m}$ (center) and $0.55 \mu \mathrm{m}$ (right), for galactic latitudes $\mathrm{b}=0,20$ and $90^{\circ}$ from top to bottom. Good seeing conditions at Cerro Paranal. Courtesy of M. Le Louarn.

us to write:

$$
n(\lambda, P, T)-1=f(\lambda) \times g(P, T)
$$

where $\lambda$ is the wavelength, and $P$ and $T$ are respectively the temperature and the pressure of the atmosphere. Taking the derivative of Eq. 28 with respect to $\lambda_{i}$ leads to :

$$
\Delta n /(n-1)=\Delta F(\lambda) / \lambda_{i}
$$

Thus, for a wavefront function corrected for the piston at a given point $x$ in the telescope pupil we get :

$$
\Gamma\left(\lambda_{i}\right)=\frac{n-1}{\Delta n} \frac{1}{\Delta \Gamma(\lambda, x)}
$$

This shows that the wavefront at any wavelength is proportional to its variation between two arbitrary wavelengths. The proportionality factor is the inverse ratio of the variation of the air refraction index between these wavelengths with respect to its value at the wavelength of interest, $\lambda_{i}$. This property applies to any quantity derived from the wavefront using a linear operator, for example: the tilt, $\theta$. Then, one gets the fundamental relation of the polychromatic LGS

$$
\theta_{\lambda_{3}}=\Delta \theta_{\lambda_{1}, \lambda_{2}}\left(n_{\lambda_{3}}-1\right) / \Delta n_{\lambda_{1}, \lambda_{2}} .
$$

Equation 31 means that, from the differential tilt measured between two wavelengths $\Delta \theta_{\lambda_{1}, \lambda_{2}}$, one can derive the tilt itself without any natural guide star. From Fig. 10, $\lambda_{1}$ has to be as short as possible, since the variation of $n$ is the steepest in the ultraviolet. 


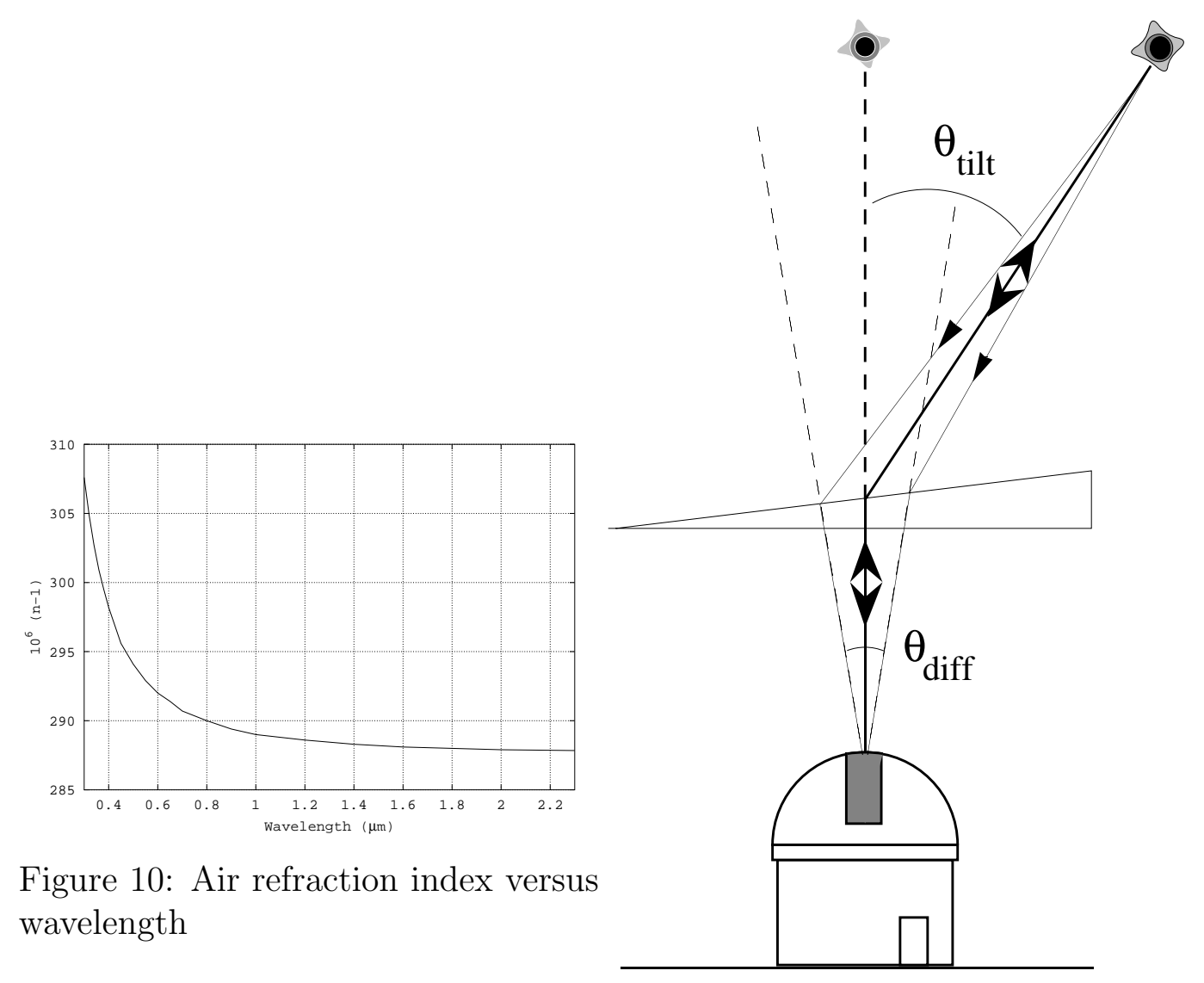

Figure 11: The emitted beam is tilted on its way to the mesosphere. It produces a spectrum spanning from $330 \mathrm{~nm}$ to $2 \mu \mathrm{m}$. The backscattered light follows different optical paths, depending on the wavelength in this spectrum. The resulting differential tilt is proportional to the tilt.

The required laser power is a function of the accuracy to be achieved in the measurements of the tilt. If it is a fraction $f$ of the Airy disk at the wavelength the tilt has to be corrected, then the allowable error in $\Delta \theta_{\lambda_{1}, \lambda_{2}}$ can be written as:

$$
\sigma_{\Delta \theta_{\lambda_{1}, \lambda_{2}}}=\frac{1.22}{f} \frac{\lambda_{c}}{D} \frac{\Delta n_{\lambda_{1}, \lambda_{2}}}{n_{c}-1}
$$


Setting it equal to the error in the measurement leads to the flux requirement:

$$
N=9.410^{-17} f^{2} \frac{\lambda_{r}^{-2 / 5}}{\lambda_{c}^{2}}\left(\frac{D}{r_{0}}\right)^{2}\left(\frac{n-1}{\Delta n}\right)^{2} .
$$

taking into account only photon noise, and assuming a simple center of gravity method to measure the spot locations (Foy et al. 1995).

How much time $t_{i}$ can we integrate the return signal to get $N$ ? The answer to this question drives the determination of the laser power required. It depends both on the temporal frequency of the tilt component of the wavefront $f_{T}$, and on the temporal ( $3 \mathrm{~dB}$ ) frequency of the closed-loop correction system $f_{3 d B}$. Parenti \& Sasiela (1994) write:

$$
f_{3 d B}=12^{1 / 4} \cdot\left(2 \pi t_{i}\right)^{-1} .
$$

Using the expression for the bandwidth error given by Tyler (1994):

$$
\sigma_{b w}^{2}=\left(f_{T} / f_{3 d B}\right)^{2}\left(\lambda_{c} / D_{r}\right)^{2}
$$

one gets:

$$
\sigma_{b w}^{2}=\frac{4 \pi^{2}}{\sqrt{12}} f_{T}^{2} t_{i}^{2}\left(\frac{\lambda_{c}}{D_{r}}\right)^{2} .
$$

with the so-called Greenwood frequency for the tilt $f_{T}=\tau_{0, t i l t}{ }^{-1}$ (see 14). Thus $t_{i}$ has to be as long as possible to decrease photon noise, and as short as possible to decrease the closed loop bandwidth error. One shows that the optimum value is:

$$
t_{i}=0.35\left[\left(\frac{n_{\lambda_{3}}-1}{\Delta n_{\lambda_{1}, \lambda_{2}}}\right)^{2} \frac{A^{2}}{N \lambda_{c}^{2} f_{T}^{2}}\right]^{1 / 3}
$$

where $A$ is the laser spot size in the mesosphere (Schöck et al. 2002).

\subsubsection{The two-photon excitation of mesospheric sodium}

Rayleigh scattering and the associated Raman scattering can provide a twowavelength LGS, e.g. a beam at 350nm can produce backscattered light at 350 and $380 \mathrm{~nm}$. The short wavelength basis leads to an unfavorably high value of the ratio $\frac{n-1}{\Delta n} \approx 125$. In addition Rayleigh scattering suffers the low altitude of the backscattering source (see Section 5). 
The most promising process to create a polychromatic LGS is the excitation of high energy levels of sodium atoms in the mesosphere. Figure 12 displays a simplified energy level diagram of the sodium electron valence. The excitation from the ground state of the $4 P_{3 / 2}$ energy level is followed

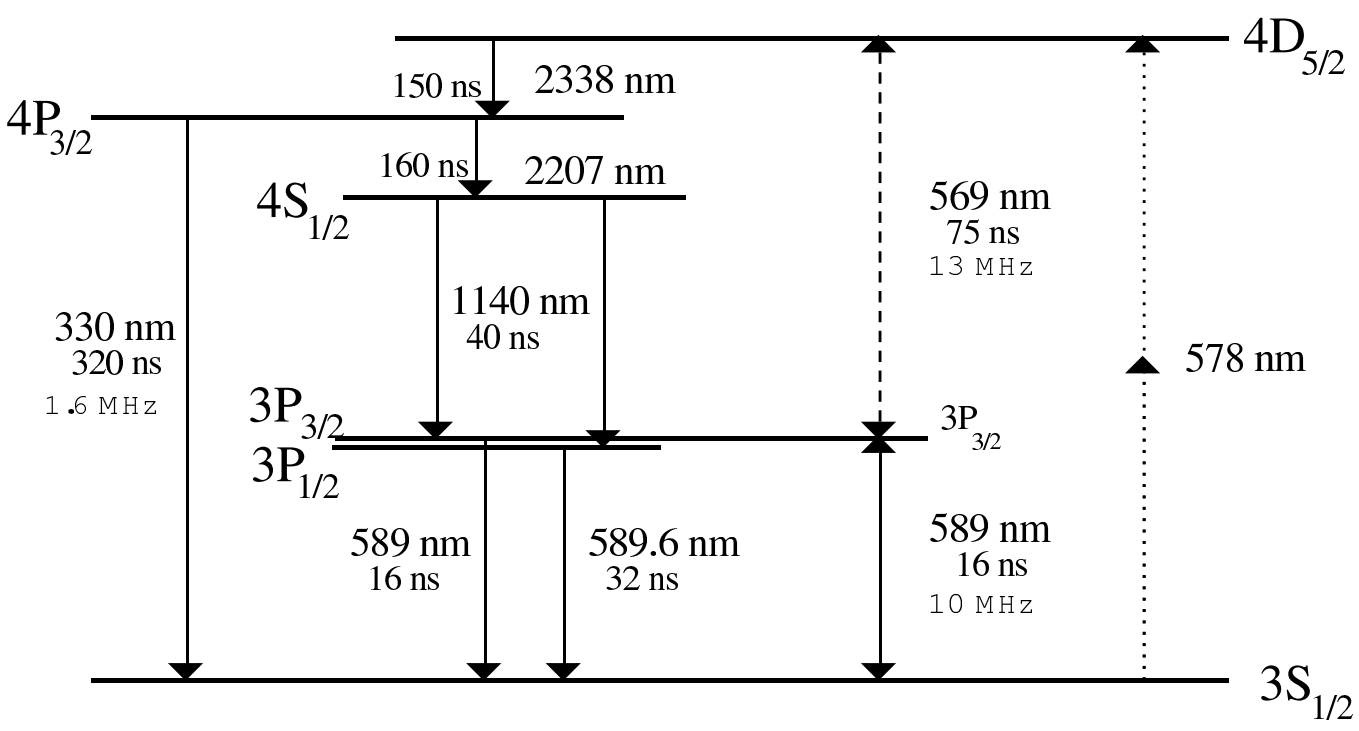

Figure 12: Energy levels of neutral sodium atom, with wavelengths (nm), lifetimes (ns) and homogeneous widths $(\mathrm{MHz})$.

by a radiative decay down to the ground state either directly or through the $4 S_{1 / 2}$ and $3 P$ levels. The backscattered spectrum ranges from $330 \mathrm{~nm}$ to $2.2 \mu \mathrm{m}$, which nicely fits the spectral coverage required for the PLGS.

This excitation can be achieved using three processes:

- Resonant one-photon excitation : The low probability of the $3 S_{1 / 2} \rightarrow$ $4 P_{3 / 2}$ transition leads to an absorption:

$$
A \lesssim \rho \sigma_{330} \Delta \nu_{H} / \Delta \nu_{T} \approx 1.6 \times 10^{-3}
$$

Taking into account the double pass of light through the comparatively poor transmission atmosphere at 330nm, and the risk of fluorescence and scattering in the innermost atmosphere, this excitation process has not been considered.

\section{- Resonant non-coherent two-photon excitation :}


The $4 P_{3 / 2}$ level can be more efficiently populated through the $4 D_{5 / 2}$ level, which decays to the the $4 P_{3 / 2}$ one emitting at $2.3 \mu \mathrm{m}$. The $4 D_{5 / 2}$ level is excited by a resonant non-coherent two-photon process with two laser beams locked on the wavelengths of the $3 S_{1 / 2} \rightarrow 3 P_{3 / 2}$ and $3 P_{3 / 2} \rightarrow 4 D_{5 / 2}$ transitions at $589 \mathrm{~nm}$ ( $D_{2}$ sodium line) and $569 \mathrm{~nm}$ respectively. Both beams must be colinear within an error negligible with respect to the size of the monochromatic spot in the mesosphere ; it has to take into account for the differential propagation of the two beams due to atmospheric refraction, which amounts to 0.055". Also in the case of pulsed lasers, pulses from each beam have to be synchronous with an accuracy significantly shorter than the mean lifetime of the $3 P_{3 / 2}$ level, which is 16 ns.

- Non-resonant coherent two-photon excitation : High energy picosecond pulses can excite the direct non-resonant two-photon absorption, involving a virtual transition at $578 \mathrm{~nm}$. This second-order nonlinear process (fast adiabatic passage (Gastineau 1995)) has a very low efficiency. First tests in the lab have been carried out at the CollisionsAgrégats-Réactivité Lab. (de Beauvoir and Pique, private communications). This process would require huge average laser powers (Biegert, Diels, \& Milonni 2000)(Pique, private communication).

Non-resonant coherent two-photon excitation can also be operated close to the resonance. Biegert, Diels, \& Milonni (2000) and independently Froc et al. (2000) have investigated the theory of this excitation, already suggested in Foy et al. (1995). Again average laser powers required at the present time to get a full population inversion do not seem compatible with a routine operation at an astronomical telescope.

As long as possible, one should avoid saturating the absorption in the mesosphere, in order to have the most efficient use of the laser power. The maximum absorption in a column occurs when the cross sections of Na atoms perfectly cover the beam section, i.e when $\sigma^{-1}$ photons are absorbed per atom. It leads to the saturation power for a velocity class of sodium atoms defined by the homogeneous width of the transition:

$$
P_{s a t_{589}}=h \nu_{589} /\left(\sigma_{589} \tau_{589}\right) \approx 185 \mathrm{~W} \cdot \mathrm{m}^{-2},
$$

where the 589 index refers to the first transition in the process, where $h$ is the Planck function, and $\sigma=1.14 \times 10^{-9} \mathrm{~cm}^{2}$ and $\tau=16 \mathrm{~ns}$ are the cross 


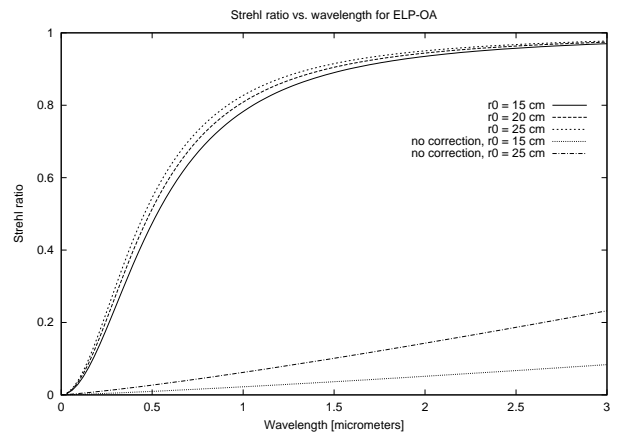

Figure 13: Strehl ratio versus wavelength for values of $r_{0}$ ranging from 15 to $25 \mathrm{~cm}$ for an 8-m telescope and measuring the differential tilt using a phase retrieval algorithm. Also shown, the Strehl ratio without any correction for values of $r_{0}$ of 15 and $25 \mathrm{~cm}$.

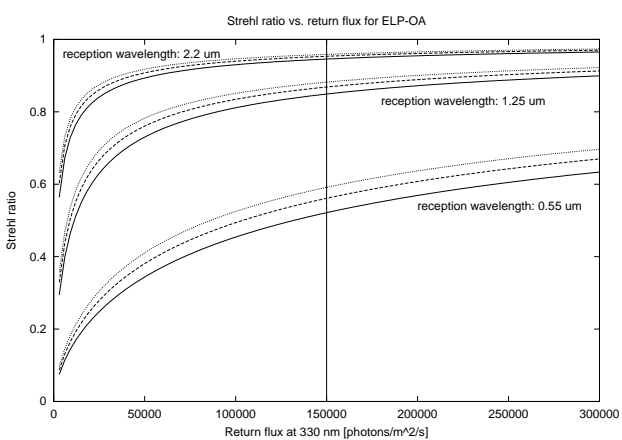

Figure 14: Strehl ratio versus return flux at $330 \mathrm{~nm}$ for an 8-m telescope (see text). From top to bottom: correction at $2.2 \mu \mathrm{m}$, $1.25 \mu \mathrm{m}$ and $0.55 \mu \mathrm{m}$. The 3 curves in each set correspond to different values of $r_{0}: 15 \mathrm{~cm}$ (solid lines), 20 $\mathrm{cm}$ (dashed lines), and $25 \mathrm{~cm}$ (dotted lines). The return flux used is normalized to an atmospheric and instrument transmission $\eta=1$. The vertical line indicates the case shown in Fig. 13.

section and the lifetime relevant to this transition. Taking into account both the hyperfine structure components of the $D_{2}$ transition, the homogeneous width $\Delta \nu_{H} \approx 10 \mathrm{MHz}$ and the Doppler width $\Delta \nu_{D} \approx 0 \mathrm{GHz}$ of each leads to the maximum absorption in the $D_{2}$ line:

$$
A_{589}=\rho_{N a} \sigma_{589} \Delta \nu_{H} / \Delta \nu_{D} \approx 2.8 \% .
$$

Similarly, one estimates the saturation power for the second transition : $P_{\text {sat }_{569}} \approx 475 \mathrm{~W} \cdot \mathrm{m}^{-2}$

A decrease by a factor of up to $\left(D / r_{0}\right)^{2}$ in the variance of the tilt measurement is expected replacing the center of gravity method by a phase retrieval algorithm (Vaillant, Thiébaut, \& Tallon 2000). It leads to the prediction of the Strehl ratios due to the tilt shown in Fig. 13 and 14.

Experiments on the sky have been carried out in order to check the efficiency of the excitation process. The first one has used the AVLIS pro- 
gramme copper vapor lasers at the Lawrence Livermore National Laboratory (Foy et al. 2000). The linearly polarized laser beams were directed toward the sky close to a natural star observed simultaneously in order to provide a reference for calibrating the return flux. Relatively high peak laser power of $\approx 2 \times 220 \mathrm{~kW}$ at a pulse repetition rate of $12.9 \mathrm{kHz}$ and $\approx 2 \times 660 \mathrm{~kW}$ at $4.3 \mathrm{kHz}$ per beam have been investigated in this experiment, as well as the balance between the powers in the 589 and $569 \mathrm{~nm}$ beams, and the spectral widening of the $589 \mathrm{~nm}$ beam (either 1 or $3 \mathrm{GHz}$, to span the $3 S_{1 / 2} F=2 \rightarrow 3 P_{3 / 2}$ or the whole $3 S_{1 / 2} \rightarrow 3 P_{3 / 2}$ transition (Fig.15). The number of return photons at $330 \mathrm{~nm}$ reaches up to $5.510^{5} / \mathrm{m}^{2} / \mathrm{s}$, which fits the requirements derived in 4.2 , but with laser powers which can hardly be installed at an astronomical telescope.

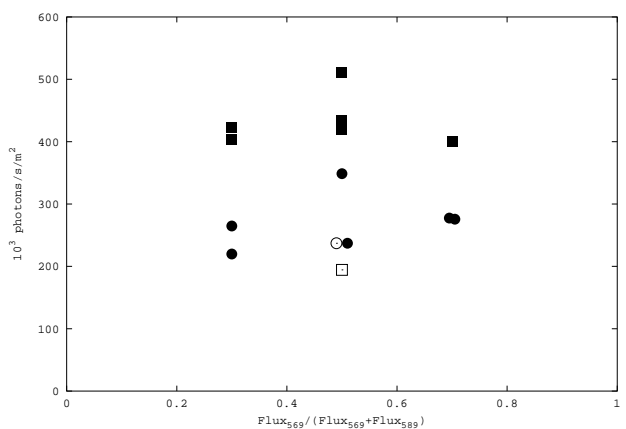

Figure 15: Two-photon excitation of the mesospheric sodium with the AVLIS cvl at LLNL. Returned flux at $330 \mathrm{~nm}$ as a function of the power balance between the two beams at $589 \mathrm{~nm}$ and at $569 \mathrm{~nm}$. Circles: modulator width at $D_{2}=1 \mathrm{GHz}$. Squares: modulator width at $D_{2}=3 \mathrm{GHz}$. Filled symbols: rep rate $=4.3 \mathrm{kHz}$; open symbols: rep rate $=12.9 \mathrm{kHz}$.

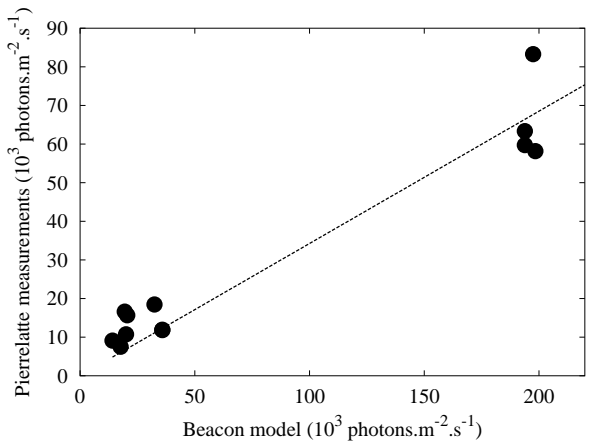

Figure 16: Return flux at 330nm: comparison between the measurements at CEA/Pierrelatte and the prediction of the CEA's BEACON Bloch optics equation model.

The second experience has used the copper vapor lasers of the SILVA programme of CEA at Pierrelatte (France), the French counterpart of the US AVLIS one. It aimed at investigating significantly lower peak powers, ranging from 10 to $90 \mathrm{~kW} / \mathrm{m}^{2}$ at the mesosphere (average power at the launch 
telescope: 10 to $100 \mathrm{~W})$. Spectral jitter shifts the wavelength of the $569 \mathrm{~nm}$ beam with respect to that of the $589 \mathrm{~nm}$ one. It results in a lower efficiency of the two-photon excitation. This effect has been investigated by amplifying a cw $1 \mathrm{MHz}$ wide single-mode dye laser in a single-stage, multipass preamplifier based on a free flowing dye jet as amplifying medium (Laubscher, Segonds, \& Pique 2000).

Taking into account that in both experiments at LLNL and at CEA the modulation function to widen the laser spectral profile are different, and that there is an uncertainty in the sodium column density, both experiments reasonably agree. They also agree with predictions from a model of the population of sodium levels based on Bloch equations (Bellenger 2002) (Fig. $16)$.

The efficiency of the two-photon excitation can be significantly improved if one increases the saturation threshold. A huge gain, by a factor at least 10, is expected if the laser spectral profile fit the Doppler line profiles, using a modeless laser (Schöck et al. 2002; Ewart 1985; Pique \& Farinotti 2003b; Pique \& Farinotti 2003a). Other increases are expected by decreasing the laser duty cycle (higher repetition rate or/and longer pulses) and by circularly polarizing the beams (Jeys et al. 1992). Finally, let us assume we shrink the laser spot in the mesosphere with an adaptive optics precompensating the outgoing laser beam. The measurement accuracy is to increased proportionally; thus, either at given Strehl ratio, the laser power can be decreased or at given laser power, the Strehl ratio is increased.

A demonstrator of a polychromatic LGS coupled with an adaptive optics is being studied to be tested at Observatoire de Haute-Provence.

Cons The PLGS concept relies on the measurement of a differential effect. Thus requirements for the return flux are higher than it would be using a first order method. It results in quite high average laser powers, with the constraint of avoiding saturation of the absorption in the mesosphere. Furthermore, two laser chains are necessary, providing two colinear, synchronous beams each of them being accurately locked on its own frequency.

We note however that the power required for each of the two beams is expected to be similar to that of the monochromatic LGS at the Keck (Wizinowich et al. 1998). 
Pros The PLGS concept provides a $100 \%$ sky coverage, likely down to visible wavelengths, without any NGS.

\subsection{The perspective method}

Mesospheric sodium atoms excited at the $3 P_{3 / 2}$ level scatter light in every direction. The backscattered beam from an auxiliary telescope $B$ meters away from the main one looks like a plume strip with an angular length:

$$
\phi \approx B \delta h / H^{2}
$$

where $\tau$ stands for the thickness of the sodium layer in the mesosphere.

The tilt can be measured from the deviation of the mesospheric plume with respect to a foreground NGS at the focus of an auxiliary telescope located in such a way that the NGS lies within the isoplanatic patch $\epsilon$ of any portion of the plume (see Fig. 17)(Ragazzoni, Esposito, \& Marchetti 1995). The tilt of the wavefront incoming onto the auxiliary telescope equally affects the plume and the NGS, as well as any image displacement due to the auxiliary telescope. There is no need to independently measure telescope jitter. Of course, two auxiliary telescopes in two perpendicular directions as seen from the main telescope are necessary to measure both components of the wavefront slope.

Only the fraction $\phi / \epsilon$ of the plume can be used to measure one component of the tilt. It results in a flux limitation, taking into account also the saturation of the absorption of the laser light by $\mathrm{Na}$ atoms. From preliminary studies, the telescope diameter of the auxiliary telescopes should range around $\approx 25 \mathrm{~cm}$ (Ragazzoni, Esposito, \& Marchetti 1995).

This concept should allow us to get a sky coverage close to $100 \%$, provided that the auxiliary telescopes are movable across a very large baseline.

\subsubsection{Technique and first experiment}

Experiments to estimate the performances of the Perspective Method have been carried out (Esposito et al. 2000) at the ALFA Laser Adaptive Optics system at the Calar Alto 3.5m German telescope in Spain (Davies et al. 2000). The laser beam was projected by the $3.5 \mathrm{~m}$ telescope and observed by a $2.2 \mathrm{~m}$ telescope $261 \mathrm{~m}$ away (Fig. 18 ). Thus the laser plume was $\approx 70$ " long. In these experiments, the $2.2 \mathrm{~m}$ telescope acts as the auxiliary telescope, and 


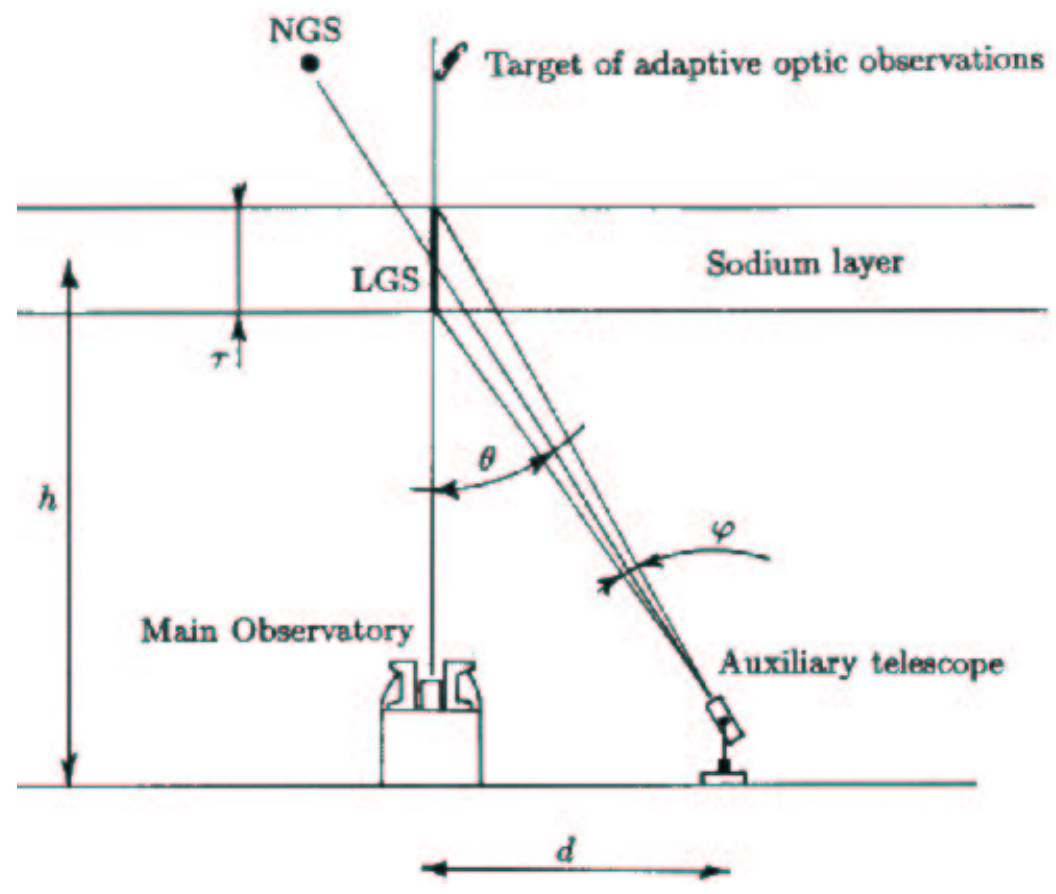

Figure 17: Principle of tilt measurement from a movable auxiliary telescope looking at a foreground NGS tracked within the isoplanatic patch of the laser plume in the mesosphere((Ragazzoni, Esposito, \& Marchetti 1995)). The telescope optical axis has to track the diurnal rotation, and simultaneously to move on the ground to keep aligned the NGS and the LGS plume. 
it is not movable. Thus close distances between the plume and NGSs occur during short time intervals. The tests have consisted of measuring the tilt

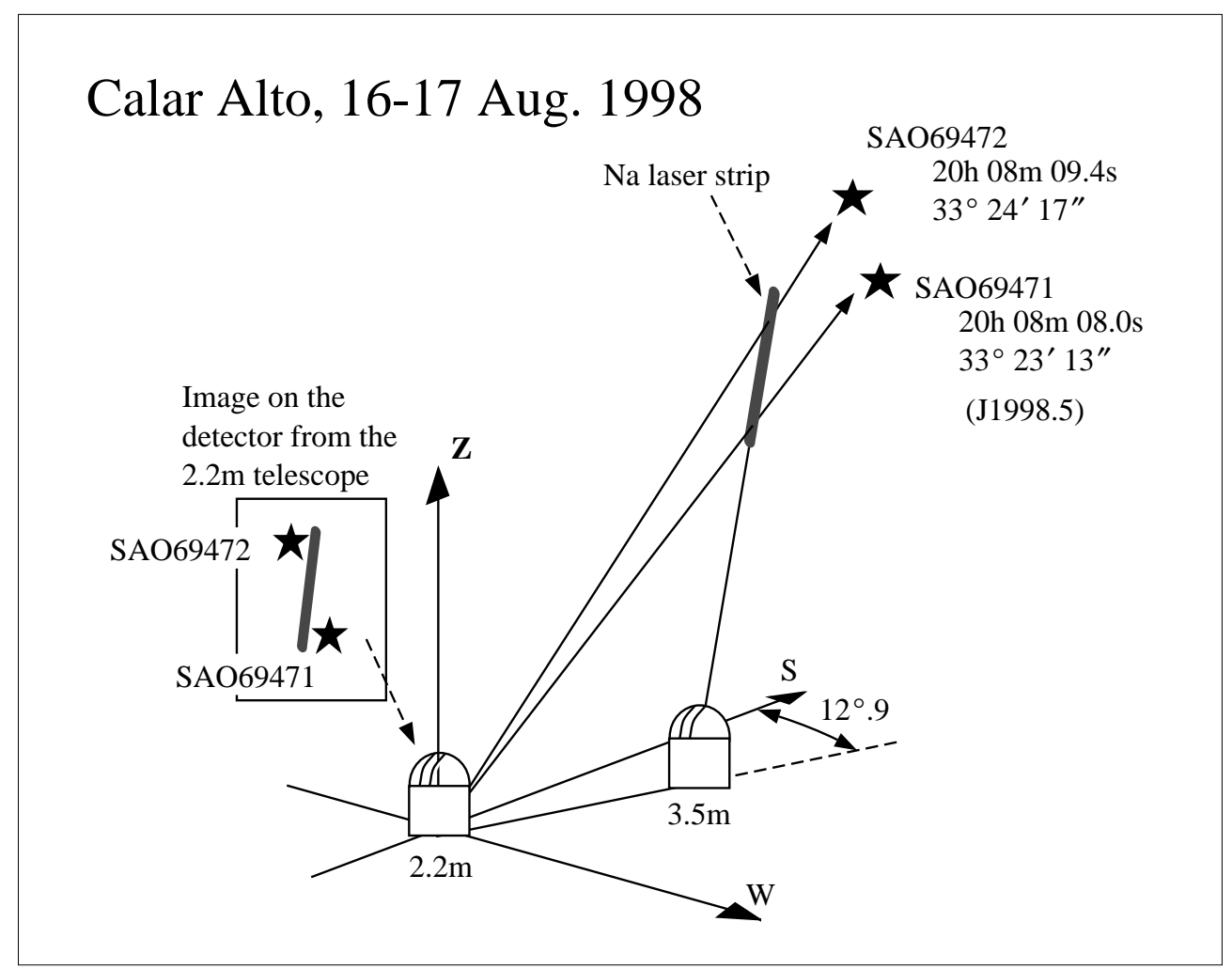

Figure 18: Arrangements of the laser projector (3.5m telescope) and observing telescope (2.2m telescope) in the measurement configuration at Calar Alto.

of two NGSs, $T_{N 1}$ and $T_{N 2}$ close to the two plume portions, $T_{L 1}$ and $T_{L 2}$ (Fig.19). A long-exposure (10s) frame is shown in Fig.19 as an example. One can compare the tilt of the NGSs with its estimate, $\tilde{T}_{N 1}$

$$
\tilde{T}_{N 1}=T_{L 1}-\left(T_{L 2}-T_{N 2}\right)
$$

\subsubsection{Technique verification and tilt estimation}

(Esposito et al. 2000) have checked the performances of the perspective method in the following way. It has been shown in (Ragazzoni 1997) that 


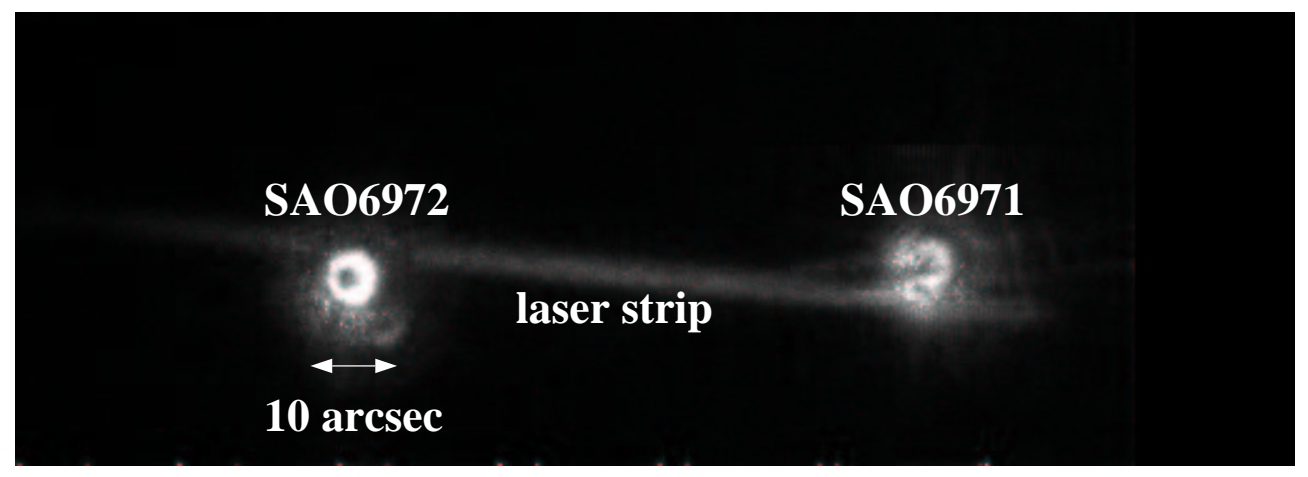

Figure 19: A MAMA subframe of $512 \times 180$ pixels at the Calar Alto $2.2 \mathrm{~m}$ telescope. Two NGSs are closely aligned with the laser plume backscattered in the mesosphere from the laser beam launched by the $3.5 \mathrm{~m}$ telescope $\approx 300 \mathrm{~m}$ away. The integration time is $10 \mathrm{~s}$, with the MAMA photon counting detector. The scale is $0.3^{\prime \prime}$ per pixel.)

neglecting the tilt component of the cone effect we have:

$$
T_{N 1}=T_{L 1}-\left(T_{L 2}-T_{N 2}\right) .
$$

To check the method, one has to measure the four tilts in Eq. 43. To evaluate the image displacement, we chose to determine the energy distribution median. It is relatively less sensitive than barycenter methods to photon noise far from the image center (Stone 1989). Data have been bined to get an integration time of 0.1 s and calculated various signals, considering a running mean with a time-width going from $0.2 \mathrm{~s}$ to $2 \mathrm{~s}$. The performance of the technique was evaluated by calculating the variances $\sigma_{1}^{2}, \sigma_{2}^{2}$ and $\sigma_{3}^{2}$ of the observables $S_{1}, S_{2}$ and $S_{3}$, so defined:

$$
\begin{aligned}
& S_{1}=T_{N 1} \\
& S_{2}=T_{N 1}-T_{N 2} \\
& S_{3}=T_{N 1}-\tilde{T}_{N 1},
\end{aligned}
$$

These variances allow us to obtain the correlation coefficients between the two pairs of tilt measurements $S_{2}$ and $S_{3}$. This is done by considering that the correlation coefficient $\gamma$ between two parameters $a$ and $b \gamma$ is related to the variances $\sigma_{a}^{2}, \sigma_{b}^{2}$ and $\sigma_{a-b}^{2}$ by the relation:

$$
\left\langle(a-b)^{2}\right\rangle=2\left(\frac{<a^{2}>+<b^{2}>}{2}\right)(1-\gamma),
$$


where

$$
\gamma=2 \frac{<a b>}{<a^{2}>+<b^{2}>} .
$$

Determinations of $\sigma_{1}^{2}, \sigma_{2}^{2}$ and $\sigma_{3}^{2}$ and of the two correlation coefficients $\gamma_{2}$ and $\gamma_{3}$ are as shown in fig. 20 as a function of the width of running mean window. However, geometrical arrangements of the NGSs with respect to the laser plume allow us to get the two tilts $T_{L 1}$ and $T_{L 2}$ via an interpolation process. This introduces an

error $\sigma_{\epsilon}^{2}$ in the $T_{N 1}$ estimate that does not pertain to the method. Thus the estimate of $\sigma_{3}^{2} \gamma_{3}$ has to be corrected for this error introduced in the

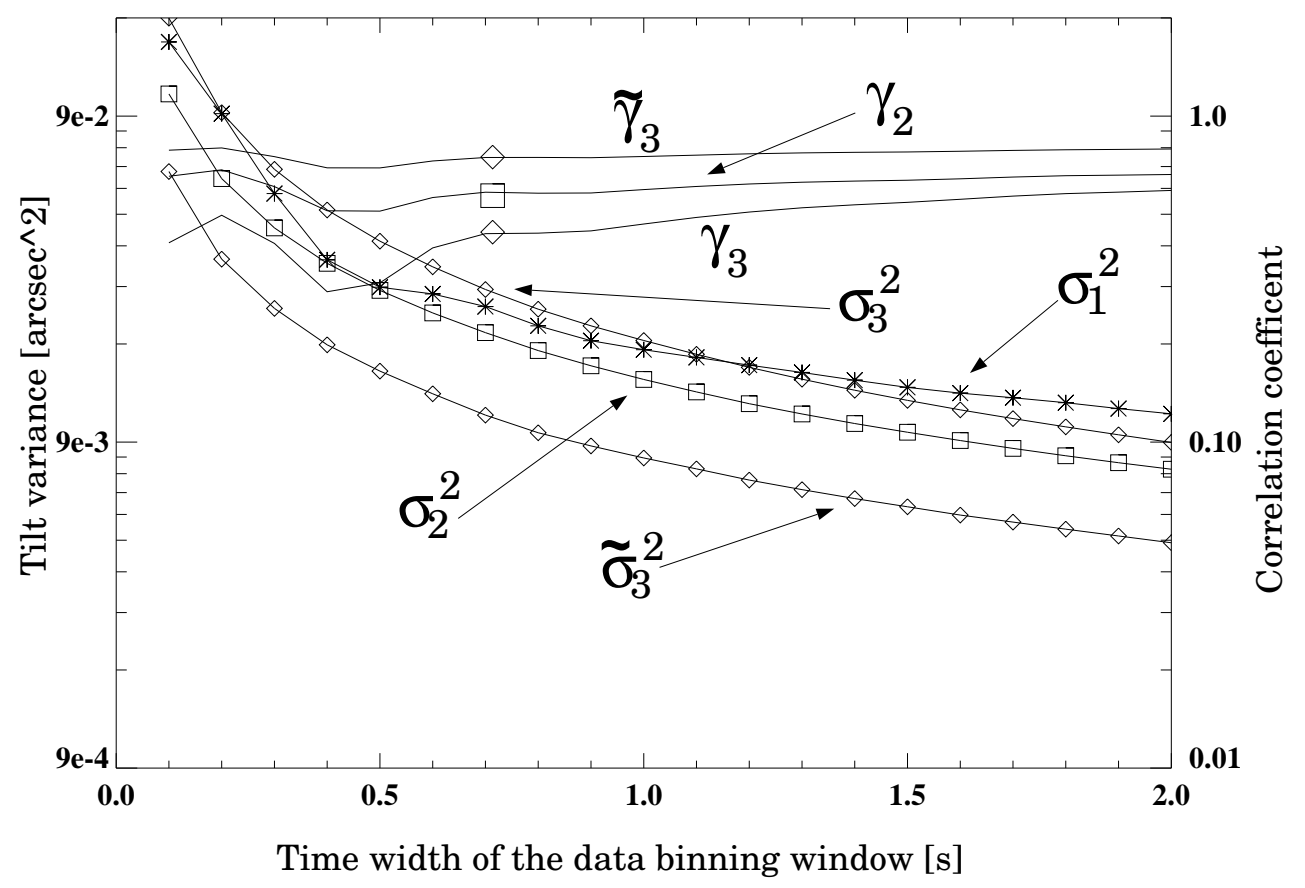

Figure 20: Behavior of the signals variance vs. the running mean window width. The continuous lines represent the correlation coefficients.

two tilts, $T_{L 1}$ and $T_{L 2}$, due to the interpolation process. Assuming that $\sigma_{\epsilon}^{2}$ is uncorrelated with the other tilt determination errors, one obtains a new variance $\tilde{\sigma}_{3}^{2}$

$$
\tilde{\sigma}_{3}^{2}=\sigma_{3}^{2}-2 \sigma_{\epsilon}^{2} .
$$

¿From $\tilde{\sigma}_{3}^{2}$ a new correlation coefficient $\tilde{\gamma}_{3}$ is derived. It is free from interpolation errors. Fig. 20 shows that the $\tilde{\gamma}_{3}$ coefficient value is $\approx 0.8$, which 
corresponds to a tilt variance reduction by a factor of 2.5 . From

$$
S_{\text {tilt }}=\left(1+\frac{\pi^{2}}{2} \sigma_{\text {tilt }}^{2}\left(\frac{D_{r}}{\lambda_{c}}\right)^{2}\right)^{-1},
$$

(Sandler et al. 1994), it would result in an improvement by up to the same factor for the Strehl ratio in the case where it is low $(\lesssim 0.1)$. Thus the method looks like promising.

Cons Two $\approx 2 \mathrm{~m}$ auxiliary telescopes moves when tracking on a surface $\approx 400 \mathrm{~m}$ in diameter around the main telescope.

The small isoplanatic patch in the visible range presumably constrains the sky coverage at these wavelengths; this has not yet been investigated.

Pros There is no need for a special laser equipment other than the monochromatic sodium laser which has to equip the main telescope for high order corrections.

\section{The cone effect}

The LGS is located in the Earth's atmosphere, in the $15-20 \mathrm{~km}$ altitude range by Rayleigh scattering, or in the $90-100 \mathrm{~km}$ altitude range, in the mesosphere. These are finite distance from the telescope as compared with the almost infinite distances of astronomical sources. It results in the socalled cone effect, or focus anisoplanatism. The volume of the atmosphere from the LGS to the telescope mirror is a cone, whereas it is a cylinder from any astrophysical source. In other words, spherical propagation cannot be neglected in the case of the LGS. This difference causes errors in the measurement of the phase disturbances of the incoming wavefronts (Foy \& Labeyrie 1985). Figure 21 shows a not to scale description of the laser beam propagating from the LGS to the telescope pupil, and the beam from the programme object. For the purpose of clarity, the turbulence is assumed to be located within two thin layers, which is quite realistic for good astronomical sites (e.g.: (Vernin \& Mũnoz-Tuñón 1994; Tallon, Foy, \& Vernin 1992b)) as shown in Fig. 1.

At the level of a turbulent layer, the annulus between the cross section of the conical beam from the LGS and the cylindrical one from the natural 


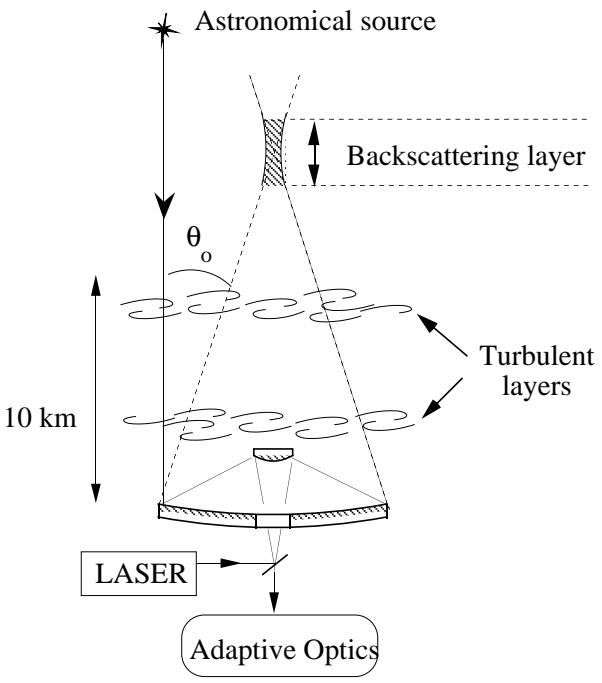

Figure 21: Propagation from the laser guide star at finite distance and from the astrophysical source at infinity to the telescope pupil through two turbulent layers. Turbulent layers are not fully sampled by the beam from the LGS, and within the sampled areas, there is a zoom effect with respect to the pupil plane. Courtesy of M. Tallon.

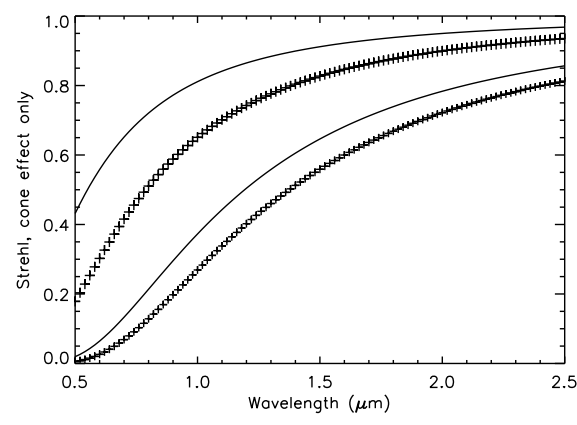

Figure 22: Strehl ratio due to the only cone effect, as a function of wavelength. Full and dashed lines: $8 \mathrm{~m}$ and $3.6 \mathrm{~m}$ telescopes respectively. Upper and lower curves: optimistic model $\left(r_{0}=25 \mathrm{~cm}\right)$ and standard model $\left(r_{0}=15 \mathrm{~cm}\right)$ for Cerro Paranal. Courtesy of M. Le Louarn 
source is not sampled on the wavefront sensor. In addition, beams from the two sources hit the same area of the pupil, where the local wavefront is sensed, after having traveled through different areas of the turbulent layers. It means that they have undergone different phase perturbations. Ignoring these differences leads to apply wrong corrections to the conjugated annulus on the AO deformable mirror. Therefore the cone effect can be considered as resulting from the anisoplanatism error between the LGS and the programme object as seen from off-axis points of the pupil: the smaller the anisoplanatism error, the smaller the cone effect.

The phase error due to the cone effect is:

$$
\sigma_{\phi}^{2}=\frac{2 \pi^{2}}{\lambda^{2}} \frac{1}{H \cos z} \int_{0}^{H} C_{n}^{2}(h) h^{2}\left(h^{1 / 3}-1\right) d h
$$

It is rather straightforward from Fig. 21 that the strength of the cone effect increases with the telescope diameter, the altitude $h$ of the backscattering layer, the zenith distance and the wavenumber of the observation. The integral shows the role not only of the integrated $C_{n}^{2}$ but of its vertical distribution because of the weighting function $h^{2}\left(h^{1 / 3}-1\right)$. It has been shown that the cone effect at large telescopes makes the image to be corrected worst than if no AO is running. Thus the cone effect has definitely to be corrected for.

Fried and Belscher(Fried \& Belscher 1994) have introduced the $d_{0}$ parameter to measure $\sigma_{\phi}$. They defined it from the phase error on the telescope pupil due only to the cone effect

$$
\sigma_{\phi}=\left(D / d_{0}\right)^{5 / 6}
$$

Thus $d_{0}$ is the telescope diameter such as $\sigma_{\phi}^{2}=1 \mathrm{rad}^{2}$.

Figure 22 shows the degradation of the Strehl ratio due to the cone effect solely. It is assumed that the deformable mirror is perfect and that there is no noise or bias; therefore the Strehl ratio should be 1 in all cases. Solid lines and dashed lines respectively refer to a $3.6 \mathrm{~m}$ and an $8 \mathrm{~m}$ telescope. Upper and lower curves have been computed with two seeing models (Viard, Hubin, \& Le Louarn 2000) at Cerro Paranal: a standard one $\left(r_{0}=15 \mathrm{~cm}\right.$ and $d_{0}=$ $3 \mathrm{~m})$ and a good one $\left(r_{0}=25 \mathrm{~cm}\right.$ and $\left.d_{0}=5 \mathrm{~m}\right)$. The figure emphasizes the importance of the cone effect; for average seeing conditions, the cone effect should be corrected even in the $\mathrm{K}$ band $(2.2 \mu \mathrm{m})$. At shorter wavelengths, the effect is still larger, as expected; even under the very best seeing conditions, the degradation factor increases from $\approx 0.8$ at $1.25 \mu \mathrm{m}$ to 0.5 at $0.55 \mu \mathrm{m}$. 


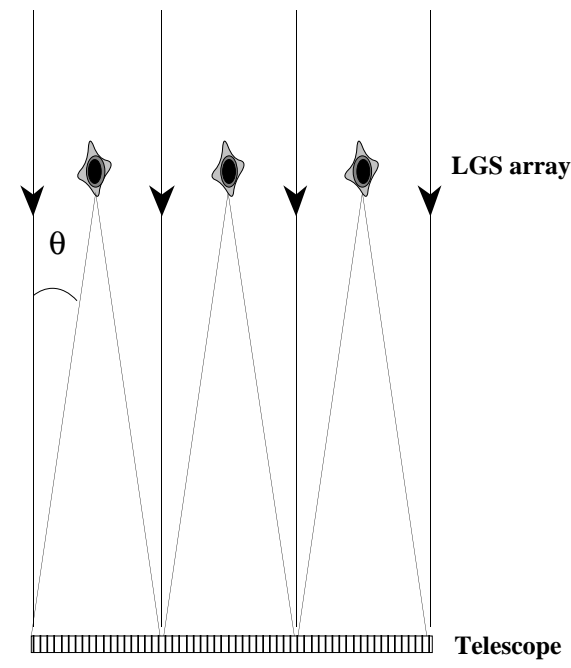

(a)

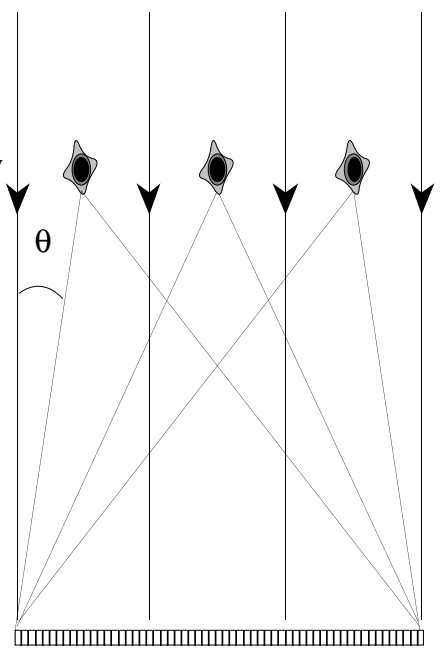

(b)

Figure 23: Two methods to process the signals from an array of LGSs. (a) The stitching method: the pupil is divided into sections. Each observes the LGS in the array in front of it, and the signal is processed independently of the other areas. (b) 3-D mapping or tomography method. Each of the pupil sections measures the whole array and the signal is processed globally (Tallon 1997).

Several ways have been investigated to correct for the cone effect. All of them rely on the use of multiple LGSs. These methods provide in the same time a significant widening of the field of view with respect to isoplanatic patch. Thus their interest is very strong in astrophysics, since objects are often larger than this field, as e.g. galaxies and star forming regions. We will shortly review these methods in the following.

\subsection{Stitching methods}

In stitching methods, one splits the telescope pupil image into as many as necessary subpupils so that for each of them the cone effect is negligible at the observation wavelength (Fig.23). A wavefront sensor per subpupil analyses the incoming wavefront. Thus, for each of them the cone effect is negligible as long as $d_{0}($ subpupil $) \approx D$. Then one boots the subpupils to restore the full pupil. As far as we know, this method has been implemented for the 
first time at the Air Force Maui Optical Site (AMOS) atop Mt Haleakala (Maui,Hawaii) by the Lincoln Lab. (Zollars 1992). The stitching method face the problem that the tilt is not known for each of the subpupils. Thus LGSs are irregularly spaced and the geometry of the LGS array is not known. It results in a fundamental limitation in the wavefront variance measurements: in case of an unrealistic infinitely dense array of LGSs, the gain in $d_{0}$ reaches the maximum factor of 2.5. (Fried 1995) if the LGS array altitude is $90 \mathrm{~km}$. This author has determined that in the case of a 6-7 spots array -the minimum size of a realistic array- and each of them being on the optical axis of a subpupil, then the gain is close to the 2.5 theoretical limiting factor.

\subsection{Tomography or 3-D mapping of the phase error}

The most promising approach is the 3-dimensional mapping of the turbulent volume in the atmosphere around the telescope optical axis (Tallon, Foy, \& Blazit 1988; Tallon \& Foy 1990; Johnston \& Welsh 1994). It is sometimes also called merging method, and more and more tomography. The basic principle compared to that of the stitching method is schematized in Fig.23.

Let us assume a LGS array. Let us consider a small area in a turbulent layer above the telescope at coordinates $(X, Y)$. Each LGS indexed $i$ will produce the same phase signal $\delta \phi$. But locations $\left(x_{i}, y_{i}\right)$ onto the telescope pupil will be different, depending on the direction of the LGS $i$ and on the layer altitude. It is somewhat similar to the principle of the SCIDAR method to determine the altitude of the turbulent layers (Vernin \& Azouit 1983) from the correlation of the wavefronts from the two components of a binary star. Let us shift the wavefront signals from the LGSs so that the

given phase signals $\delta \phi$ originating from $(X, Y)$ add. The same shifts will cause phase signals from other locations in the turbulent layer to average. In this way the map of the turbulent volume above the telescope can be restored, thus allowing to command several deformable mirrors, each of them conjugated of a turbulent layer (Fig. 24, or of an atmosphere slab (Beckers 1988). This author proposed to call the principle of an adaptive optics with several deformable mirrors multiconjugate adaptive optics, now referred in literature as MCAO. He proposed also to use the term of atmospheric tomography for the 3-D measurements of the phase variance. In fact it is not really tomography since each LGS senses a different volume, and since the indetermination of the tilt for each turbulent layer leads to a degenerescence of the inverse problem to solve. This method of measuring the 3 -D distribution of 


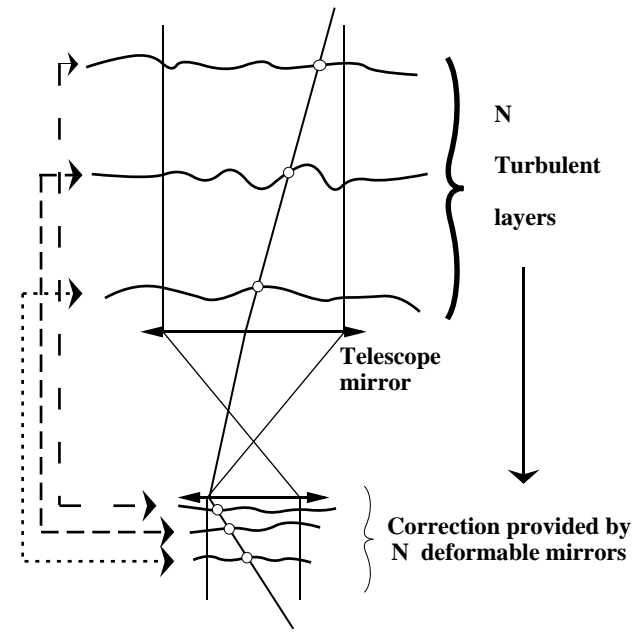

Figure 24: The principle of multiconjugate adaptive optics. Here, there are three turbulent layers; an optics device forms conjugated images of these three layers onto three deformable mirrors. Each of these mirrors is fed with the phase error signals in the conjugated layer. For the purpose of clarity, a field lens at the telescope focus is not shown.

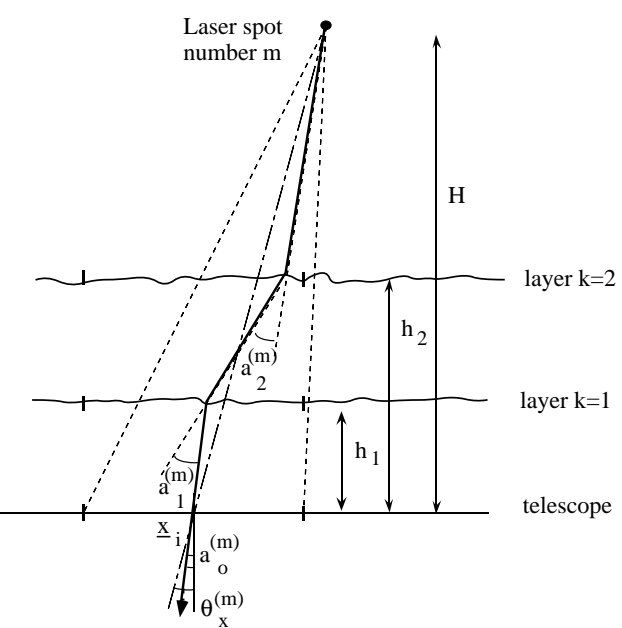

Figure 25: Propagation of a beam through the turbulent layers down to the telescope pupil. See text for approximations and notations.

the phase variance has never been implemented.

It is worthwhile noting here that the first attempt to measure phase errors in the volume above the telescope and to propose the concept of MCAO (without giving it a special name) had been proposed in the case of natural guide stars for adaptive optics (Dicke 1975). This author assumed that the turbulence vertical profile can be described with only two turbulent layers, located respectively at low and high altitudes. The low component was assumed quasi-stationary, and propagation was assumed to have negligible effects on the phase pattern, under moderate seeing conditions ( $\lesssim 2 \operatorname{arcsec})$. Taylors' hypothesis (Taylor 1938) of a phase screen moved by wind was assumed for the upper turbulent layer. Thus the wind velocity $v$ moves by its dimension a $r_{0}$ blob in $r_{0} / v$. The phase complex amplitude is moved at the same velocity on the telescope pupil. Therefore combining the phase distri- 
butions observed at the pupil with a time lag of $r_{0} / v$ leads the determination of both the high and the low distributions of phase. This method uses temporally separated observations of a single NGS, as faint as magnitude 11. It would provide a significant improvement in the Strehl ratio over a field up to $\approx 2$ arc minutes. It has never been implemented, as far as we know.

The 3D-mapping or tomography can be described as follows. Let us consider an array of LGSs at the altitude $H$ and at locations $\left(X_{\mu \nu}, Y_{\mu \nu}\right)$. It is observed with a Shack-Hartmann type wave front sensor with $N$ subpupils with central coordinates $\left(X_{i j}, Y_{i j}\right)$ in the pupil plane. Thus it provides $2 \times N$ measurements of the slope of the incoming wavefront. They measure the $N 2$-D phase delays encountered by the wavefront crossing the $m$ turbulent layers. Measurements and phase delays are related by a matrix equation which has to be inverted. This is similar to tomography problems with the additional problem that the equation array is degenerated because of the tilt indetermination, as we will see below. To write the propagation of a beam from a LGS to the telescope so that equations are linear, one needs the following assumptions.

- Discrete turbulence : : Turbulence is concentrated in $m$ thin layers at distance $h_{m}$ from the telescope. Typically $m$ is 2 or 3 . This is consistent with SCIDAR (Vernin \& Azouit 1983) campaigns to measure the vertical distribution of $C_{n}^{2}(h)$ (Vernin \& Azouit 1983) or balloon (Bufton et al. 1972; Vernin \& Mũnoz-Tuñón 1994), as shown in Fig.1 . It shows a typical $C_{N}^{2}(h)$ profile, which is strong mostly within three thin layers: they contribute for $\frac{\int_{\text {lower boundary }}^{\text {upper bound } C_{N}^{2}(h) d h}}{\int_{0}^{\infty} C_{N}^{2}(h) d h}=33 \%, 32 \%$, and $13 \%$ respectively to the total seeing. $\approx 80 \%$ of the seeing is produced by only three thin layers.

- Geometric propagation : approximation works (scintillation is negligible). It implies : $\lambda / r_{0} \leq r_{0} \times \cos z \times h_{n}^{-1} \forall n$ where $n$ is the number of the thin turbulent layer and $h_{n}$ its altitude. At zenith, assuming that the altitude of the highest turbulent layer is $8 \mathrm{~km}$ above the telescope, at $\lambda=550 \mathrm{~nm}$ it yields the lower limit $r_{0}>7 \mathrm{~cm}$. Thus it is a realistic condition for good astronomical sites.

- Small perturbation approximation : : it means that a ray crossing the turbulent layer from a laser spot toward a point of the pupil does not depart significantly from a straight line, i.e.: it crosses each turbulent 
layer in the same coherence area as the straight line from the laser spot to that point of the pupil. Thus the optical path fluctuations caused by the turbulent layers simply add independently

(Roddier 1981). This approximation is considered as valid for zenith distance $z \lesssim 60^{\circ}$ (T.Young 1970).

With simple geometry one can write the location of a beam associated with the $(\mathrm{i}, \mathrm{j})$ subpupil above the $m^{t h}$ turbulent layer when it propagates along the $z$ axis (Tallon \& Foy 1990):

$$
\begin{aligned}
& \vec{P}_{i j}^{m}(z)= \\
& \quad\left(X_{i j}+\left(z-h_{m}\right)\left(\sum_{k=0}^{m} a_{p q}^{(k)}+\sum_{l=1}^{m}\left(h_{l}-h_{l-1}\right) \sum_{k=0}^{l-1} a_{p q}^{(k)}\right)\right) \vec{x} \\
& \quad+\left(Y_{i j}+\left(z-h_{m}\right)\left(\sum_{k=0}^{m} b_{p q}^{(k)}+\sum_{l=1}^{m}\left(h_{l}-h_{l-1}\right) \sum_{k=0}^{l-1} b_{p q}^{(k)}\right)\right) \vec{y}
\end{aligned}
$$

The $\left(a_{p q}, b_{p q}\right)$ are the changes in the slope of the wavefront at $(\mathrm{p}, \mathrm{q})$. Writing Eq. 51 for all the subpupils yields to the array:

$$
\left\{\begin{array}{l}
X_{\mu \nu}-X_{i j}-H a_{i j}^{(0)}=\sum_{k=1}^{m}\left(H-h_{k}\right) a_{p q}^{(k)} \\
Y_{\mu \nu}-Y_{i j}-H b_{i j}^{(0)}=\sum_{k=1}^{m}\left(H-h_{k}\right) b_{p q}^{(k)}
\end{array}\right.
$$

The left hand terms of Eq. 5.2 are the departures from the unperturbed propagation measured by the wavefront sensor at the telescope. The right hand terms are the sum of the deflections when the rays cross the turbulent layers are weighted by $\left(1-\frac{h_{k}}{H}\right)$. It can be shown that this system is not stable, because the global tilt cannot be measured; the tilt for at least one of the turbulent layers has to be set to 0 ; it has to be measured from some other way (see 4).

This description can be written closer to that of the command of the deformable mirrors, considering the propagation from the LGSs array to the pupil (Tallon, Foy, \& Vernin 1992b; Tallon, Foy, \& Vernin 1992a; Le Louarn \& Tallon 2000; Le Louarn \& Tallon 2002). Let us consider the laser spot at angular position $\theta_{\mu \nu}$. At the telescope, the phase corrugation the wavefront 
from this LGS undergoes when crossing a turbulent layer at altitude $h_{m}$ is magnified by a factor $\gamma_{m}=H /\left(H-h_{m}\right)$. In fact the operator describing the propagation can be developed in:

- a parallel propagation along the path $h_{m} / \gamma_{m}$,

- a magnification by a factor of $\gamma_{m}$, and

- a shift of the image of $\gamma_{m} \theta_{\mu \nu} h_{m}$

Let be $\overrightarrow{\psi_{m}}$ the vector gathering the samples of the layer $m$. After propagation to the pupil along $h_{m} / \gamma_{m}$, it writes as

$$
\overrightarrow{\Phi_{m}}=\mathbf{P}_{m}\left(\overrightarrow{\psi_{m}}\right)
$$

where $\mathbf{P}_{m}$ is a linear operator, due to the small perturbation approximation.

The magnification changes $\vec{\Phi}_{m}$ to $\Gamma_{m} \vec{\Phi}_{m}$, with $\Gamma_{m}=1, \gamma_{m}^{-1}$, or $\gamma_{m}^{-2}$ respectively if the $\overrightarrow{\Phi_{m}}$ gathers the phases, the slopes or the curvatures. The magnified and shifted sampling grid projected onto the pupil does not coincide with the sampling of the wavefront sensor. One has to resample it, through a linear combination of the $\vec{\Phi}_{m}$ components: $\vec{\Phi}_{m}^{\mu \nu}=G_{m}^{\mu \nu} \Gamma_{m} \vec{\Phi}_{m}$.

Adding the contribution of each layer yields the general equation array:

$$
\vec{M}^{(\mu \nu)}=\sum_{k=1}^{m} G_{k}^{(\mu \nu)} \Gamma_{k} \vec{\Phi}_{k}
$$

where $\vec{M}^{(\mu \nu)}$ is the vector of measurements for the LGS $\mu \nu$.

There are as many equations 52 as turbulent layers, and as many equations 53 as LGSs in the spot array.

Thus we get $2 N$ equations per laser spot $(\mu, \nu)$. The number of unknowns is $2 \mathrm{Nm}$. If the inversion is well conditioned, then one needs as many laser spots as turbulent layers. One can show that there is at least a case where the matrix cannot be

inverted: that of an array of 2 spots observed with a 1-dimensional pupil through 2 turbulent layers (Tallon \& Foy 1990). In fact the rank of the matrix is too small by 1 , so that an extra condition is required to constrain the inversion. This condition may be:

$$
\sum_{p} \sum_{q} a_{p q}^{(k)}=0 \text { and } \sum_{p} \sum_{q} b_{p q}^{(k)}=0
$$

which results again from the fact that the global tilt is unknown, and that we should not attempt to measure it from the array of monochromatic LGSs. 


\subsection{Modal approach}

A modal approach has also been proposed (Ragazzoni, Marchetti, \& Rigaut 1999), which should be easier to implement, to allow us filtering and handling of practical situation such as the telescope central obscuration.

Several extended studies of multiconjugate adaptive optics have been developed, e.g.:(Johnston \& Welsh 1994; Ellerbroek 1994; Tokovinin, Le Louarn, \& Sarazin 2000; Le Louarn \& Tallon 2002). It is beyond the scope of this review to discuss about these methods.

\section{$6 \quad$ Lasers for the laser guide star at telescopes}

Laser characteristics depend on the physical process involved to create the LGS. The quality of a laser beam is given in general by its $M^{2}$ :

$$
M^{2}=\pi * \theta * d /(2 / \lambda)
$$

where $\theta$ is the full angle of the beam. The choice of the laser beam diameter $d$ is not trivial. It depends on the quality of the laser beam (characterized by its $M^{2}$ ), of the quality of the astronomical site (characterized by $r_{0}$ ) and of the optimal diameter for the artificial star. Two extreme cases are to be considered. If $d \lll r_{0}$, then the shape of the laser spot in the mesosphere is dominated by the beam $M^{2}$. On the other hand if $r 0 \lll d$, then it is dominated by atmospheric turbulence. For a given $r_{0}$ and $M^{2}$, there is an optimum $d$. But it is not easy to maintain it. Indeed, generally, $M^{2}>1$ and it increases with laser power. Moreover given an astronomical site, $r_{0}$ fluctuates in the short term. But whatever this process, a common requirement is that the beam quality must be better than the seeing disk (typically $0.2 \mu$ radian) defined as $\lambda / r_{0}$ corresponding to $\sigma_{\phi}^{2}=1$ radian across $r_{0}$. If the variance of the beam is fixed to a tenth of the variance of the phase errors due to the atmospheric turbulence, then the beam quality should be better than $1 / 9 \times d / r_{0}$ in diffraction limit unit. At the European VLT, typically $r_{0} \approx 15$ $\mathrm{cm}$ and the choice of the beam diameter made by ESO is $36 \mathrm{~cm}$ at $e^{-2}$ and a projector diameter of $50 \mathrm{~cm}$ (to ensure the magic ratio $d / r_{0} \approx 3$ ). Because of these conditions $M^{2} \approx 1$. A diameter of $25 \mathrm{~cm}$ would be compatible with $M^{2} \approx 1.4$. This could relax a lot laser technology difficulties, at the expense of a laser spot in the mesosphere twofold larger. High power laser beams with $M^{2} \approx 1$ require adaptive optics at the emission. 


\subsection{Lasers for Rayleigh backscattering}

A definite constraint for lasers in the case of Rayleigh backscattering is that they must be pulsed lasers. Indeed in this case a temporal gate selects backscattered photons from a slab in the atmosphere. The slab thickness is $\approx 1 \mathrm{~km}$, because at higher values the spot elongation becomes not negligible as view from the edge of the telescope mirror (see 41 in 4.3). Thus backscattered photons are received during $2 / 300000=6.7 \mu \mathrm{s}$, the factor 2 coming from the round trip time from the lower to the upper level of the slab. The detector should not receive photons from any other slab during this time interval, as it would be the case with a cw laser. If we consider that Rayleigh scatter is significant up to the altitude of $30 \mathrm{~km}$, the pulse repetition rate must be lower than $3 \times 10^{5} / 30 / 2=5 \mathrm{kHz}$, and the pulse length must be shorter than $6.7 \mu$ s to avoid contamination of the photon flux from the selected slab from photons backscattered at other altitudes.

The first observation in open literature of a LGS created by range gating Rayleigh backscattering in the $10-15 \mathrm{~km}$ altitude range at $1.5 \mathrm{~m}$ telescope of the Lunar Laser Ranging Station of Observatoire de la Côte d'Azur (Séchaud et al. 1988; Foy et al. 1992). These authors investigated the properties of the LGS wavefront compared with wavefront observed for natural guide stars. A doubled YAG laser operated at $10 \mathrm{~Hz}$ with $0.3 \mathrm{~J}$ per pulse was used.

Excimer lasers are comparatively low cost devices, compact with a good wall plug efficiency. Light pollution due to scattering is located in the ultraviolet part of the spectrum, making easy to filter it, except of course for those astrophysical programmes requiring to observe at these wavelengths. Thus these laser are well suited for Rayleigh scattering. Let us mention finally another significant advantage of UV lasers: airplane plastic windows do not transmit light at $351 \mathrm{~nm}$, so that there is no operation restriction from air traffic agencies (at least in the USA). Up to now, a drawback of UV lasers is the lower quantum efficiency of CCDs in this spectral range.

Excimer lasers can easily fit the power requirement, as shown e.g. by the UnISIS developed by the University of Illinois (Urbana Champaign) at the Mount Wilson 2.5m telescope (Thompson et al. 1998). A $50 \mathrm{~W}$ excimer laser at $351 \mathrm{~nm}$ pulsed at either 167 or $333 \mathrm{~Hz}$ is focused to an altitude of $18 \mathrm{~km}$ above the site $(\approx 20 \mathrm{~km}$ above sea level). The backscattered light feeds an adaptive optics device with a Xinetics 177 actuator deformable mirror. Figure 26 shows an UnISIS LGS formed close to the star $\alpha O p h$. New high power and high repetition rate $(1 \mathrm{kHz})$ frequency tripled YAG laser at 355 
nm should also be very interesting devices for Rayleigh backscattering.

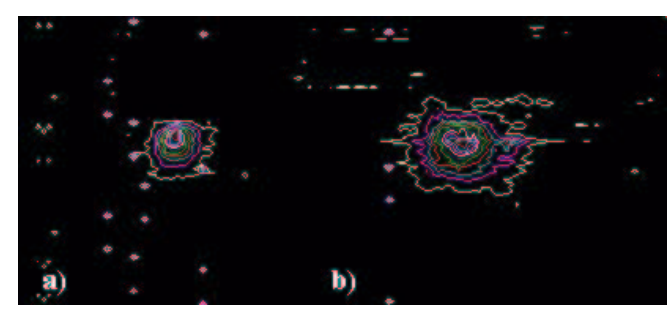

Figure 26: UNISIS Rayleigh LGS close to the NGS $\alpha O p h$. FWHMs of these images are respectively 2.3 and 1.8". As expected, the FWHM of the LGS is larger than that of the NGS because the laser beam crosses twice the turbulent atmosphere.

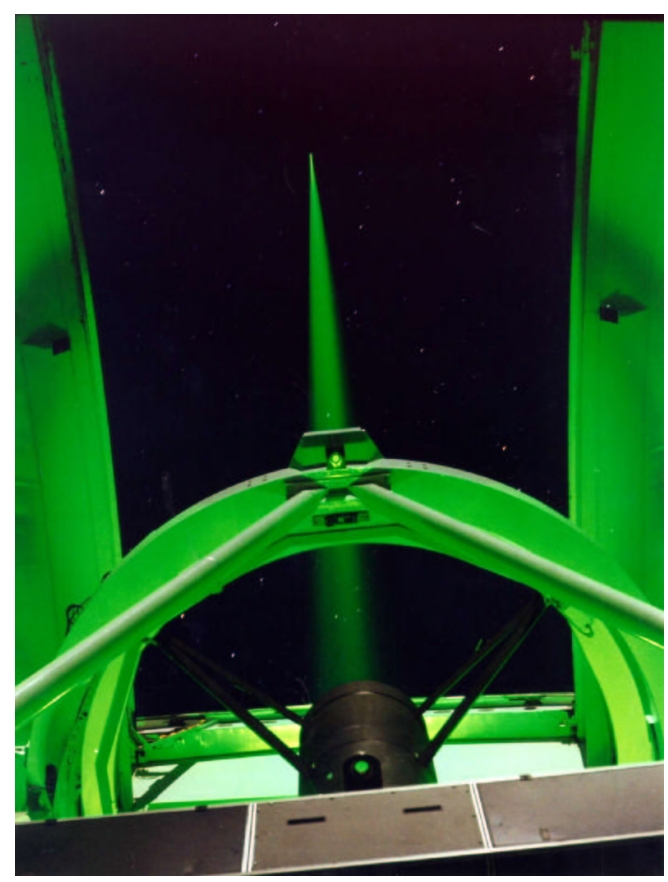

Figure 27: The laser beam emitted from the $4.2 \mathrm{~m}$ WHT telescope. The backscattered LGS is selected at $\approx 20 \mathrm{~km}$ where the beam is focused.

Rayleigh scattering has also been demonstrated at the $1.5 \mathrm{~m}$ telescope of the Starfire Optical Range of the Air Force Research Laboratory at Albuquerque (Fugate et al. 1994) with the first operation in 1989. In their second generation experiment, they used a copper vapor laser. The $150 \mathrm{~W}$ average power was equally shared between the two $\mathrm{Cu}$ lines at 510.6 and $578.2 \mathrm{~nm}$. The pulse length was $50 \mathrm{~ns}$ and the repetition rate $5 \mathrm{kHz}$. The altitude range gated for the LGS was $2.4 \mathrm{~km}$ at $10 \mathrm{~km}$. The adaptive optics had a 341 actuator deformable mirror, operated at a closed loop bandwidth of $143 \mathrm{~Hz}$. It provided a Strehl ratio $S=0.64$ when the Shack-Hartmann wavefront sensor was fed with a natural guide star, and $S=0.48$ with a LGS. As expected the LGS Strehl is lower then the NGS one. Indeed the cone effect is not corrected, and the LGS FWHM is larger than the NGS one since the beam crosses twice the turbulent atmosphere; it leads to a lower signal to noise 
ratio of the reconstructed wavefront.

Rayleigh backscattering is also chosen by University of Durham for its Restricted Conjugate adaptive optics programme. First tests occurred in 2001 at the $4.2 \mathrm{~m}$ WHT telescope. The laser is a $5 \mathrm{~W}$ frequency doubled Nd:YLF. The beam is emitted through a $30 \mathrm{~cm}$ telescope. An image of the beam launched from the telescope is shown in Fig. 27. The repetition rate of $7 \mathrm{kHz}$, which may prove to be a little bit too high to prevent two pulses to backscatter simultaneously light from altitudes lower than the altitude range selected to create the LGS, at zenith distance $z \lesssim 60^{\circ}$.

Up to now, "white" femtosecond lasers do not look like suitable for LGSs, because they cannot propagate above $\approx 10 \mathrm{~km}$ high, and because of thermal effects in the atmosphere.

\subsection{Lasers for $\mathrm{Na}$ resonant scattering}

There are two constraints for a laser device to excite mesospheric sodium atoms, which to some extent are contradictory.

The power has to be high enough to insure that there are enough return photons to measure the wavefront corrugations with enough accuracy. Modeling as well as experiments, e.g. with the ALFA system at Calar Alto, show that an average power of $\gtrsim 10 \mathrm{~W}$ at the mesosphere is required. As far as we know, up to now there is no commercial solution to produce such a power with a continuous wave laser. A strong advantage of cw lasers is that saturation of the $\mathrm{Na}$ absorption does not occur, thanks to the duty cycle equal to 1. It is quite straightforward to get these power, and even much higher ones, with pulsed lasers.

On another hand, increasing the average power of pulsed lasers obviously increases the peak power accordingly. Then arises the problem of saturation. When the absorption of the laser light by sodium atoms in the mesosphere begins to saturate as the peak power increases, the efficiency of the excitation decreases. In fact the return flux still increases thanks to power broadening as the square root of the laser power. Since avoiding any energy loss in the close environment of telescopes is a major concern, one generally considers that one should work with the highest possible plug efficiency, i.e.: below the saturation threshold (see 3.6). Since the repetition rate cannot be increased significantly above $\approx 25 \mathrm{kHz}$, the only way to quench saturation is to widen the spectral profile. Currently, phase modulators provide us with a gain by a factor of $\approx 2$ in the return flux at saturation. A significant progress has been 
very recently achieved with the modeless laser developed within the frame of the above mentioned ELP-OA polychromatic LGS programme (Pique \& Farinotti 2003b; Pique \& Farinotti 2003a). This new oscillator can widen the laser spectral profile in order to fit the Doppler $D_{2}$ line profile with a quasi gaussian profile (see the optical layout in Fig. 28. The measured gain with respect to a monochromatic beam is $\approx 5$ (which means a factor of $\approx 20$ for the two-photon excitation process involved in ELP-OA). To evaluated the gain achieved by a modeless laser a rate equation model has being used for the monochromatic LGS (see above references).

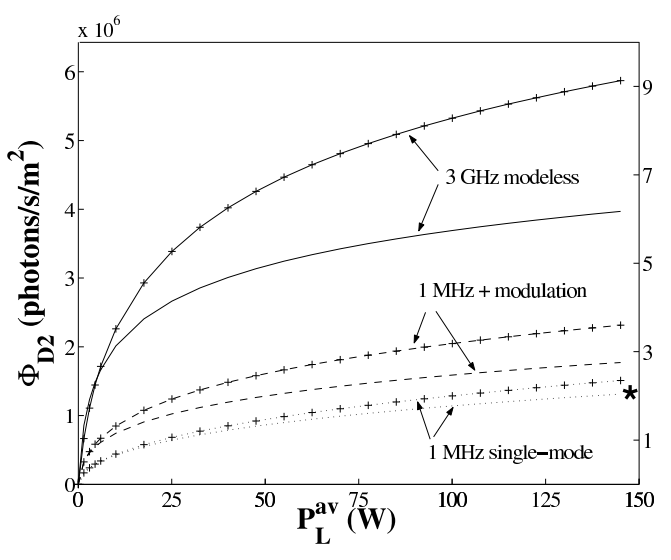

Figure 28: Return sodium fluorescence flux versus average laser power, for three types of laser: $1 \mathrm{MHz}$ single-mode laser (doted lines), 3 $\mathrm{GHz}$ and $4.5 \mathrm{GHz}$ modeless laser (dashed and solid lines respectively). The lines with and without crosses correspond respectively to: i) $80 \mathrm{~ns}$ pulse width, $30 \mathrm{kHz}$ repetition rate and ii) $40 \mathrm{~ns}$ pulse width, $15 \mathrm{kHz}$ repetition rate.

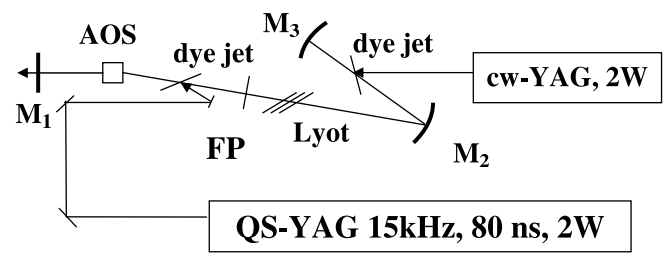

Figure 29: Modeless laser configuration. Two dye jets are pumped by a cw YAG laser and a Q-Switched YAG laser respectively. The mirrors M2-M3 are highly reflecting for R6G dye. M1 has 3\% transmission coating at $589 \mathrm{~nm}$. The acousto-optic shifter (AOS) is made of TiO2. A Lyot filter and an étalon (FP) control the linewidth and the absolute wavelength. The pre-amplifier jet is pumped by a Q-switched frequency doubled YAG laser.

This model includes spectral and temporal characteristics of lasers. Figure 28 shows the numerical results. The modeless laser returns much more photons than single mode and modulated lasers. The corresponding return flux with a $30 \mathrm{GHz}-80 \mathrm{~ns}$ laser is close of that expected from a cw laser of the same mean power. A third constraint is the wavelength. A sodium LGS 
requires a $589 \mathrm{~nm}$ laser and Na PLGS requires a second laser at $569 \mathrm{~nm}$. In the first case we will see that several solutions exist or are in the process of development. On the other hand, to date, only dye lasers can produce both the wavelengths of the sodium PLGS.

$\mathrm{Na}$ LGSs relying on cw lasers use either $\mathrm{Ar}^{+}$or Nd:YAG or similar lasers to pump a dye. When relying on pulsed lasers, the pump is a copper vapor laser or a pulsed Nd:YAG, which pumps dye master oscillators.

\subsubsection{First Na LGS experiments}

As far as we know, the first experiments of a LGS onto the sky were carried out at the 1.2m SWAT telescope of the Lincoln lab (Primmerman et al. 1991; Humphreys, Bradley, \& Herrmann 1992) and at the $1.5 \mathrm{~m}$ SOR telescope of the Air Force Research Lab (Fugate et al. 1994). The first observation in open literature of a LGS created by resonant backscattering in the mesosphere has been carried out at the $2.2 \mathrm{~m}$ telescope of the University of Hawaii at Mauna Kea (Thompson \& Gardner 1987) using cw ring dye laser. It was not basically different from lidar techniques to study the properties of the mesosphere (Megie \& Blamont 1977; Gardner et al. 1986). Also the first successful attempt to get adaptive optics images using a mesospheric sodium LGS has been carried out at the MMT (Lloyd-Hart et al. 1995); using a Spectra-Physics 380C dye laser pumped by a $20 \mathrm{~W}$ Spectra Physics $171 \mathrm{Ar}^{+}$ multiline laser, the output power was $\approx 1 \mathrm{~W}$, generating a 10.4 magnitude star; at $2.2 \mu \mathrm{m}$ the Strehl ratio was increased by a factor of $\approx 2$ and the FWHM was improved from 0.58 " for seeing limited images down to 0.42 ". The laser beam was launched coaxially with the telescope. The following experiments gave up this method because the diffusion of the laser beam by the mirrors of the telescope was too intense.

The Lincoln Lab. operated a flashlamp pumped Rhodamine 6G dye at the $1 \mathrm{~m}$ telescope of White Sands Missile Range. The energy was 40mJ/GHz, with a pulse spectral width of $3 \mathrm{GHz}$. The pulse length was $4 \mu \mathrm{ms}$ at a pulse repetition rate of $20 \mathrm{~Hz}$. The telescope both launched the laser beam and observed the backscattered light. The authors observed the Na resonant backscattering at $\approx 95 \mathrm{~km}$ and the Rayleigh backscattering from 6 to $20 \mathrm{~km}$. They studied in detail the cone effect as a function of altitude by studying the tilt difference between two subapertures of the edge of the main mirror opposite with respect to the optical axis, and comparing this difference with the same parameter simultaneously measured with a bright natural star. 
They concluded that the behavior of the cone effect with altitude fits the theoretical predictions.
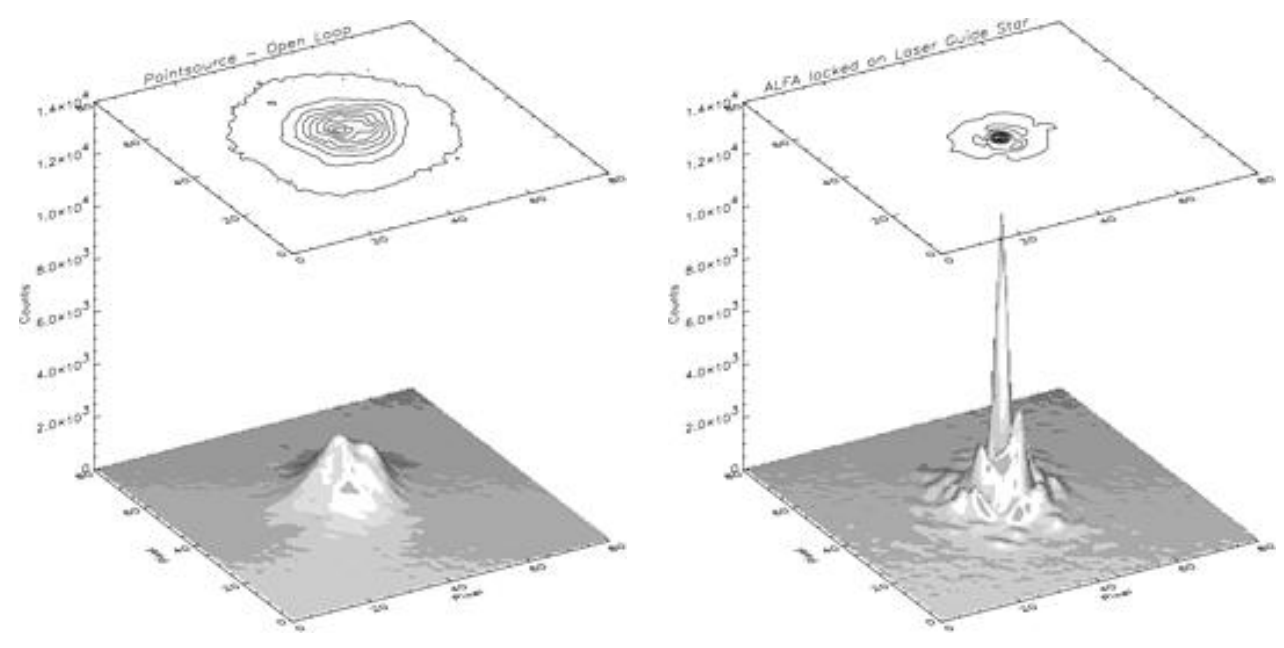

Figure 30: Two images of the unresolved star SAO 68075 at $2.2 \mu \mathrm{m}$, obtained with the ALFA adaptive optics and LGS device at the Calar Alto 3.5m German telescope. Without any correction the FWHM of a 200s exposure image is $0.65 "$. On the left, the adaptive optics improved the 200s image down to 0.19 ", with a Strehl ratio of $12 \%$. On the right, the best 10s image is shown. A Strehl of $23 \%$ was reached, and a diffraction limited core (0.14") as well as diffraction rings are clearly visible. The first 18 Karhunen Loeve modes of the incoming wavefronts were corrected by the adaptive optics device. The high-order AO loop was closed at $75 \mathrm{~Hz}$ on the LGS located 10 " away from the star. The tip-tilt was corrected on the star itself at $65 \mathrm{~Hz}$.

ALFA at Calar Alto Max Planck Institute für Extraterrestrische Physik (MPIfE) (Garching, RFA) has developed ALFA (Adaptive-optics with a Laser For Astronomy), a prototype LGS device to feed the adaptive optics system of the German 3.5m telescope at Calar Alto (Kasper et al. 2000). The AO deformable mirror had 97 ceramic piezo actuators. Wavefronts were measured by a Shack-Hartmann sensor with three different samplings of the telescope pupil: 6,18 , or 30 subpupils selected as a function of the seeing and of the reference source (NGS or LGS which has a larger FWHM). The frame rate of the Lincoln Lab CCD ranged from 25 to $1200 \mathrm{~Hz}$. 
The laser system (Rabien et al. 2000) is a Coherent 899 ring Rh6G dye laser pumped by a Coherent Innova $200 \mathrm{Ar}^{+}$. The $27 \mathrm{~W}$ multiline output of the pump was optimized to maximize the output power. A $4 \mathrm{~W}$ single-longitudinal-mode laser beam of good quality has been obtained using intracavity and extracavity servo-locking. The output spectral bandwidth was obtained with Fabry-Perot étalons and a birefringent filter; the central wavelength was tuned with a Brewster plate.

ALFA have been in operation from 1998 until 2001.

\subsubsection{Running Na LGS experiments}

The Lick Observatory LGS First LGS-aided adaptive optics images at an astronomical telescope have been obtained at Lick observatory (Max et al. 1997). It has been improved until now and it is in regular operation for astrophysical observations (Gavel et al. 2002). The wavefront sensor of its adaptive optics device is a Shack-Hartmann with an hexagonal grid 40 subapertures $43 \mathrm{~cm}$ in diameter. It is read with a $64^{2} \mathrm{LL}$ CDD with a readout noise of $6 \mathrm{e}^{-} /$pixel at $500 \mathrm{~Hz}$. The deformable mirror has 61 active actuators.

The laser technology used at Keck rises from research on the LLNL AVLIS isotopic separation programme. The LGS system relies on a dye master oscillator pumped with a flash lamp pumped frequency doubled solid state Nd:YAG. The average output power measured at the exit of the $30 \mathrm{~cm} \mathrm{di-}$ ameter launch device is $11 \mathrm{~W}$. Pulse length and rate are respectively 100 ns and $11 \mathrm{kHz}$. Whereas the pump is located in the telescope building, the dye master oscillator is attached to the side of the telescope structure. The LGS referenced adaptive optics delivers Strehl ratios as high as 0.53 at 2.2 $\mu \mathrm{m}$ using 16th magnitude close natural stars to sense the tilt.

The Keck Observatory LGS The Lick LGS has been a prototype for the development of the LGS programme at the Keck Observatory. Figure 31 show a layout of this device. The grazing incidence dye master oscillator is pumped with a 50W YAG pulsed at $26 \mathrm{kHz}$. This high repetition rate as well as the long pulse duration of $120 \mathrm{~ns}$ lead to the rather high duty cycle $310^{3}$; it increases the average power of the saturation threshold. A single mode fiber transfer the $3 \mathrm{~mW}$ beam to the preamplifier, which is also pumped with a 50 W YAG. Finally the beam is amplified with $460 \mathrm{~W}$ YAGs to deliver 16-20 W average power. Before the pre-amplifier, two phase-modulators broaden the beam spectral profile with side band lines contained in an envelope of $3 \mathrm{GHz}$ 


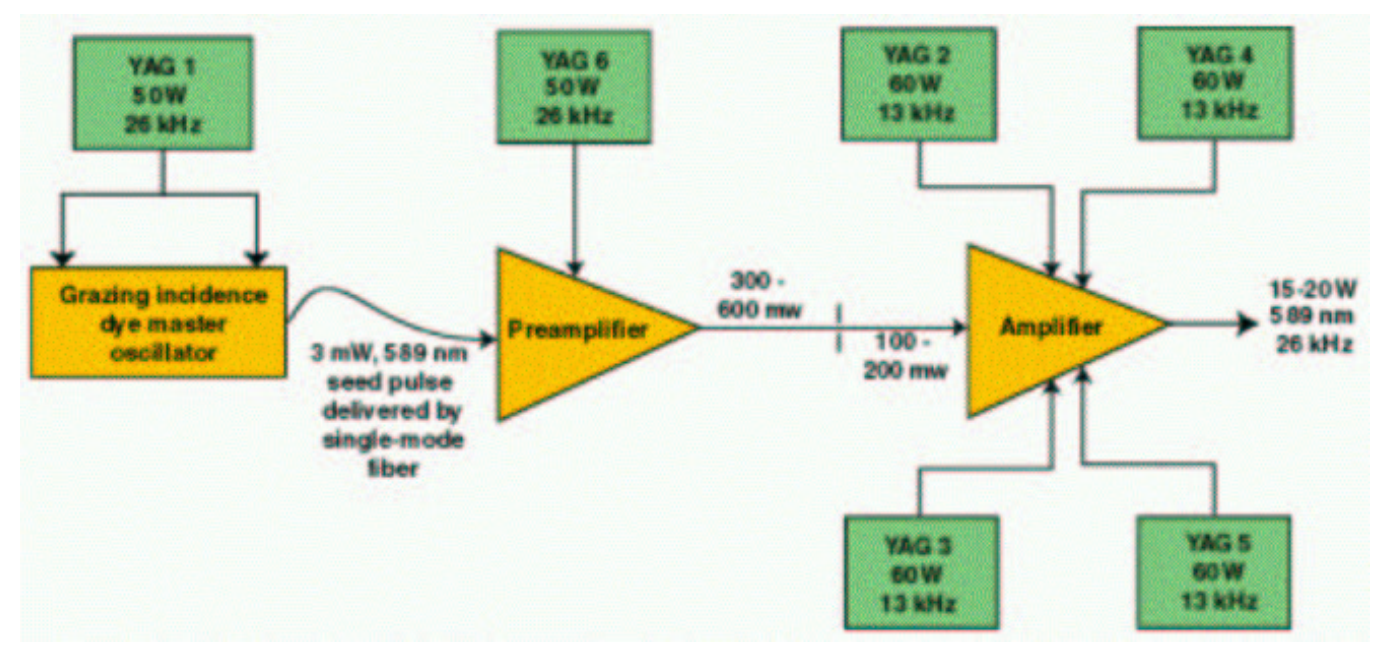

Figure 31: Scheme of the laser device to generate the sodium $\mathrm{D}_{2}$ LGS at Keck observatory

to fit the $\mathrm{D}_{2}$ line Doppler profile. The dye master oscillator and its pump are installed in a room in the telescope dome. The pre-amplifier, amplifier, the diagnostic tools and the launch $50 \mathrm{~cm}$ Gregorian telescope are attached to the main ring on the side of the telescope. The output beam is $30 \mathrm{~cm}$ in diameter. The laser channel installed at Keck is a big and complicated system with high maintenance (Contos et al. 2002).

The Keck got its first LGS light end of 2001, The coupling with the adaptive optics is scheduled for 2003 (Pennington et al. 2002a).

\subsubsection{Na LGS projects}

PARSEC: the European VLT LGS The ALFA team is preparing a LGS system for one the $48 \mathrm{~m}$ telescopes of the Very Large Telescope of the European Southern Observatory (ESO) at Cerro Paranal (Northern Chile), in collaboration with ESO. It will feed the wavefront sensor of the 256 actuators adaptive optics device NAOS (Rousset et al. 2000; Rousset et al. 2002). The beam will be launched by a $50 \mathrm{~cm}$ telescope located behind the $0.9 \mathrm{~m}$ secondary mirror of the telescope, inside the central obscuration. It minimizes the spot elongation in the Shack-Hartmann images corresponding to the edge of the pupil, with respect to the case of launching the beam from the side of the telescope mount as done for all the smallest telescopes. 


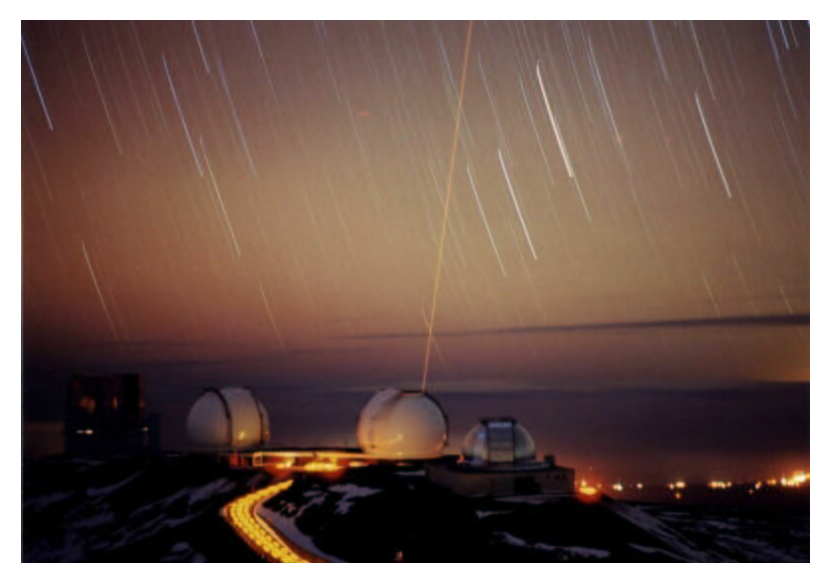

Figure 32: The laser beam emitted by the $10 \mathrm{~m}$ Keck-II telescope at the top of the $4200 \mathrm{~m}$ high Mauna Kea sleeping volcano in Hawaii. It is taken $600 \mathrm{~m}$ away, from the Canada-France-Hawaii (CFHT) telescope. One sees the faint Mie + Rayleigh scattering, as well as the star trails in the sky due to the diurnal motion of the stars during the 20 mn exposure. Hazard lights from a vehicle mark the steep descent path of the summit. Photo credit: John McDonald, CFHT Corp.

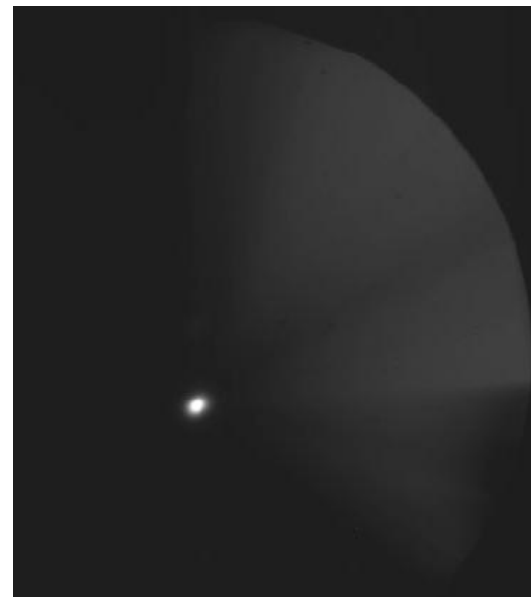

Figure 33: One of the very first spots created by the Keck-II LGS in the mesosphere. The FWHM is 1.9". The sharpest LGS image obtained at the Keck-II has a FWHM of 1.4". The diffuse area on the right half part of the image is the light pollution coming from the top of the Rayleigh backscattering at altitudes up to $50-60 \mathrm{~km}$.

The dye master oscillator is a Coherent 899-21 ring cw dye laser.It is pumped with a $10 \mathrm{~W}$ frequency doubled Nd:YAG (VERDI Coherent). The output power of this first stage is $\approx 2.5-3 \mathrm{~W}$. Then a relay optics stabilizes the direction of the beam and formats it to mode-match the amplifier cavity spatial mode. The beam is then amplified with 4 more 10W VERDI lasers to pump 2 high velocity dye-jets each on both sides. The expected output is $10 \mathrm{~W}$, whereas $12.8 \mathrm{~W}$ has already obtained at lab when pumping the jets with $48 \mathrm{~W} \mathrm{Ar}^{+}$multiline light. Thus the expected output power is 18-20 W when they will be pumped with $4 \times 10 \mathrm{~W}$ VERDI lasers at $532 \mathrm{~nm}$ (Rabien et al. 2002). The beam quality is $M^{2} \approx 1.3$. The spectral line bandwidth is $\approx 10 \mathrm{MHz}$. In fact PARSEC will produce a single frequency beam, which 
will be broadened into 4 separate lines in the beam relay system entrance to assist in transmitting the high power beam through the fiber and also to avoid saturating the sodium layer. These lines lie within an envelope 0.5 $\mathrm{GHz}$ wide to better fit the $\mathrm{D}_{2}$ line Doppler profile.

ESO plans to transfer the $10 \mathrm{~W}$ and latter on $20 \mathrm{~W}$ output beam by a mono-mode fiber to the launch telescope which is located within the telescope central obscuration, behind the secondary mirror. It uses photonic crystal fibers from Crystal Fiber AG. They have a $15 \mu$ mpure silica core. They are still being tested (Bonaccini et al. 2002).

PARSEC is expected to have its first light on the sky by the end of 2003.

ARC telescope at Sac Peak A very interesting alternative is the frequency sum laser, which trades upon a coincidence. By chance the sum of the frequencies of the two lines of two Nd:YAG lasers at 1.064591 and 1.31925 $\mu \mathrm{m}$ gives a new line at $0.589159 \mu \mathrm{m}$ which is the wavelength of the $D_{2}$ line (see Fig. 34). Thus summing these two components of a Nd:YAG output in a non linear LBO crystal produces the desired wavelength (Jeys, Brailove, \& Mooradian 1989; Jelonek et al. 1994). The power at the output of the crystal at the $\mathrm{D}_{2}$ line wavelength can be expressed as a function of the powers $P_{1.06}$ and $P_{1.32}$ at 1.06 and $1.32 \mu \mathrm{m}$ :

$$
P_{0.589}=\eta P_{1.06} P_{1.32} L^{2} \frac{\sin ^{2} \Delta k l / 2(\Delta k l / 2)^{-2}}{d^{2}}
$$

where $\eta$ and $l$ are the conversion efficiency and the length of the crystal, $d$ is the beam diameter in the crystal, and $\Delta k=k_{1.06}+k_{1.32}-k_{0.589}$, the $k$ being the wavenumbers. $l$ is limited by the walk-off phenomena. To prevent saturation of the absorption in the $\mathrm{Na}$ layer at high $P_{0.589}$, one can use a mode locked laser (Jeys, Brailove, \& Mooradian 1989). One shows that the optimum mode spacing of the laser cavity is $\approx 100 \mathrm{MHz}$, which is close to $\tau^{-1}$ where $\tau=16 \mathrm{~ns}$ is the $\mathrm{D}_{2}$ transition lifetime. The train of micropulses is absorbed as a cw laser would be during the time of the macropulse.

The sum frequency laser technique developed at the Lincoln Lab has been used for the ChAOS project at the ARC telescope (Kibblewhite et al. 1998). The Nd:YAG laser is pumped by laser diodes. Micropulses and macropulses are respectively $0.57 \mathrm{~ns}$ and $150 \mu \mathrm{s}$, the spectral bandwidth is $0.9 \mathrm{GHz}$ and the mode locked output power is $9.5 \mathrm{~W}$. With an output power of a $5 \mathrm{~W}$, the magnitude of the LGS generated in this way reach 9.2 for a 1 " spot. The Strehl ratio has been improved by a factor of 12 at $0.85 \mu \mathrm{m}$ under 1 " 


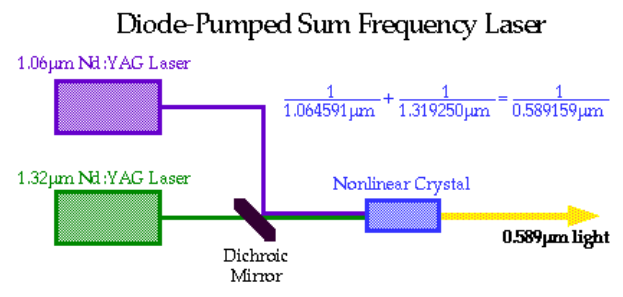

Figure 34: The superposition of the Nd:YAG beams at $1.06 \mu$ mand $1.32 \mu$ mproduces in the LBO non linear crystal a beam of which the frequency is the sum of the frequency of the two input beams. The inverse of this frequency is by chance exactly the wavelength of the sodium $D_{2}$ line at $0.589 \mathrm{~nm}$. (Courtesy of D. Pennington)

seeing conditions. Now the goal in collaboration with Lite Cycles Laser is to produce 20 to $40 \mathrm{~W}$ with $1 \mathrm{kHz}$ repetition rate in a Zig-Zag slab lasers and 3 LBO crystals. Active thermal lens control should be necessary. A $M^{2}$ of 1.5 is expected. Unfortunately there is no such favorable coincidence to produce the second $569 \mathrm{~nm}$ laser line for Na PLGS.

GEMINI The two GEMINIs $8 \mathrm{~m}$ telescopes will be equipped with sum frequency generated sodium LGSs, with a similar layout developed with Coherent Technologies Inc.(Pentland et al. 2002). The output power will be 20 $\mathrm{W}$, with $510 \mathrm{ps}$ micropulses with a $94 \mathrm{MHz}$ pulse repetition rate. As in the ESO VLT, the launch telescope is located behind the secondary mirror, but the beam is transported there by mirrors from the laser table on the side of the telescope center section. GEMINI North, at Mauna Kea (Hawaii), will be equipped with a single LGS.

An ambitious programme is running to equip the GEMINI South telescope with a multiconjugate adaptive optics device fed with an array of 5 LGSs (Ellerbroek et al. 2002). There are 3 deformable mirrors, amounting to 769 actuators and 51020 subapertures wavefront sensors, one per LGS in the array. In this project the tilt is sensed with three natural reference stars in the field. It is planed to use the same kind of laser as for GEMINI North, but improved to deliver at least $50 \mathrm{~W}$, or to use five $10 \mathrm{~W}$ lasers. A general layout of the whole system is shown in Fig. 35.

There are a few other programmes at different locations.

\section{- Starfire Optical Range :}

The Starfire Optical Range at Albuquerque (USA/NM) has successfully 


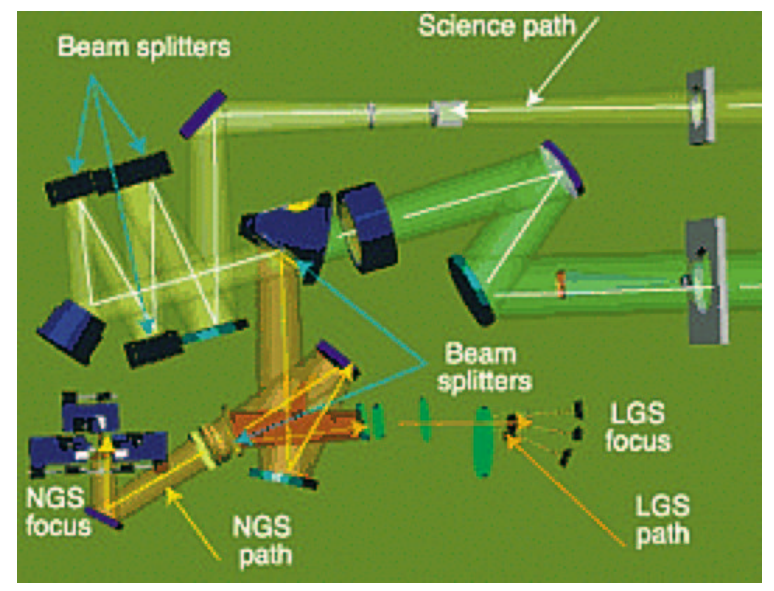

Figure 35: Layout of MCAO system with 5 LGSs for the GEMINI South $8 \mathrm{~m}$ telescope. There are 3 deformable mirrors, 5 wavefront sensors fed by 5 LGSs, and three tip-tilt sensors using natural stars.

demonstrated a $20 \mathrm{~W}$ sum frequency cw beam (Bienfang et al. 2003). A high efficiency of $55 \%$ has been obtained. It is planed to increase the output power up to $50 \mathrm{~W}$ adding a second source (Telle, Milonni, \& Fugate 2000; W.Milonni, Fugate, \& Telle 1998). This laser has produced a 7.1 magnitude guide star on the sky.

\section{- William Hershel Telescope :}

A Rayleigh guide star is planed for the NAOMI adaptive optics at the $4 \mathrm{~m}$ WH telescope (Rutten et al. 2002). The goal is to provide limited spatial resolution $(\approx 0.2 ")$ across a comparatively large field. Rayleigh scattering will produced wit a $\approx 25 \mathrm{~W}$ Nd:YLF or YAG laser focused at $15 \mathrm{~km}$; in this project it is assumed that most of the turbulence strength is located at low altitudes above the Roque de los Muchachos site. The $\approx 40 \mathrm{~cm}$ launch telescope will installed behind the secondary telescope. The project should be completed within 2.5 years from initiation.

- MMT :

An array of Rayleigh LGS is in preparation for the $6.5 \mathrm{~m}$ MMT telescope (Georges III et al. 2002). It will feed a MCAO device, so that the drawback of the low altitude of the laser spot is partly balanced. In order to increase the altitude range gated to observe the spot, a dynamic refocus system is being tested. The purpose is to keep the beam focused in a fixed plan while the pulse propagates through the atmosphere, thanks to a vibrating concave membrane mirror. As a result, the integration time per pulse can be longer, and thus the return flux collected is higher. A prototype is being experimented with an 
$1.5 \mathrm{~m}$ telescope. The laser source is a tripled Nd:YLFdiode pumped solid-state laser, delivering a beam at $351 \mathrm{~nm}$ with an $M^{2}=1.1$.

\section{$7 \quad \mathrm{R} \& \mathrm{D}$ in progress about new lasers for LGSs}

Research and development programmes are in progress in order to get more suitable lasers for LGSs. Main cases for these efforts are:

- Power : It was clear from the very first LGSs experiments and theoretical models that the limiting magnitude of the laser spot has to be $\approx 9$. It implies an (average) power larger than $15 \mathrm{~W}$. Either one needs to develop more powerful $\mathrm{cw}$ lasers, or to improve the efficiency of sum frequency lasers, or to improve the match of the laser spectral profile to the Doppler using modeless lasers (Pique \& Farinotti 2003a; Pique \& Farinotti 2003b).

- Reliability and maintenance : MTBF is a concern for laser systems to be operated routinely, out of a physics laboratory, i.e. with little maintenance facilities. Also maintenance has to be reduced at the very minimum. For instance it is a drawback of actual dye lasers, for which the dye liquid needs to be changed typically on a weekly basis. But one can easily imagine that the dye is changed like a toner of an ink-jet printer. It is only a problem of technological development.

Fiber lasers may prove to bring elegant solutions to these challenges. Fibers used for these application are doped with rare earth elements as erbium, ytterbium or germanosilicates. It is the lasing medium. Fiber Bragg gratings bound the cavity. They are pumped by injecting in one end a laser beam, generally a laser diode. The other end deliver the laser output. These devices are very compact. Since there is no liquid or chemicals, they are safe and they require a minimum maintenance with a high MTBF. The only open question is the possibility to get high powers in the fibers without damage.

The LLNL and ESO are developing together an all fiber sum frequency laser (Pennington et al. 2002b). They use a Nd:Silica fiber lasing at 0.938 $\mu \mathrm{m}$, which had to be scaled from $10 \mathrm{~mW}$ to $2.2 \mathrm{~W}$. The second laser is an Er fiber lasing at $1.583 \mu \mathrm{m}$. The beams are summed in a periodically poled crystal (PPLN or PPLT or PPKTP). The goal is to generate a $10 \mathrm{~W}$ beam at $0.589 \mu \mathrm{m}$. The erbium fiber has already produced a $10 \mathrm{~W}$ beam. 
Several methods are studied to suppress the amplified stimulated emission at $1.088 \mu \mathrm{m}$ (e.g.: cooling the core with liquid nitrogen). The PP crystals are a challenge. It is difficult to make them growing long enough. To avoid photorefractive damages, an issue is to use strongly elliptical beams, in order to reduce the intensity in the crystal. The LLNL will test this technique at $532 \mathrm{~nm}$, where PPKPT are available. The goal is to demonstrate in 2003 that $5-10 \mathrm{~W}$ can be generated, to be able then to build an integrated system.

ESO is also developing another technique relying on a Raman fiber laser pumped with a $1.1 \mu \mathrm{m} \mathrm{cw} \mathrm{Yb} \mathrm{doped} \mathrm{fiber} \mathrm{laser} \mathrm{(Hackenberg,} \mathrm{Bonaccini,}$ \& Werner 2002). It delivers a beam at $1.178 \mathrm{~nm}$ which is then frequency doubled to produce the $589 \mathrm{~nm}$ beam.

Whereas at the beginning of the LGS era the prevailing idea was that one should use available lasers to generate LGSs, it is now clear that they definitely require specific $R \& D$ programmes. One expect much more efficient LGSs, i.e.: brighter reference sources for the wavefront sensors. The theory of the correction of the cone effect and the widening of the corrected field seems now to be rather well mastered. Prototypes to test it on the sky are being built, from which the feedback is now necessary for further progress. The problem of the tilt measurement from a LGS is still open. It sometimes claimed that it will be no longer a difficulty with hectometric optical telescopes, since their diameter will be larger than the atmosphere outer scale. This is likely not true at the accuracy of the diffraction limit of the telescope. Several issues have been proposed and work is in progress to solve this problem. The dream of the astrophysicist is to get diffraction limited images at the focus of the ground based largest telescopes from the near ultraviolet to the thermal infrared, over a reasonably wide field. It is of course a challenge in particular at visible wavelength. Step by step this dream comes to reality. The success of these developments is mandatory for the efficiency of the next generation of hectometric optical telescopes, with respect to sciences cases, but also with respect with competing space observatories.

Beckers J. M., 1988, "Increasing the size of the isoplanatic patch with multiconjugate adaptive optics", in M.-H. U. (ed.), Very Large Telescopes and their instrumentation, ESO conferences No. 30, ESO, Garching, Germany, p. 693 
Bellenger V., 2002, Ph.D. thesis, Université Paris VI, Paris, France

Biegert J., Diels J.-C., Milonni P. W., 2000, "Bi-chromatic 2-photon coherent excitation of sodium to provide a dual wavelength guide star", Optics L., 25,683

Bienfang C., Denman C. A., Grime B. W., Hillman P. D., Moore G. T., Telle J. M., 2003, "20 Watt CW All-Solid-State 589-nm Sodium Beacon Excitation Source Based on Doubly Resonant Sum-Frequency Generation in LBO" ", in Solid-State Photonics, OSA Topical Meeting on Advanced, p. in press

Blamont J., Donahue T., 1961, J. Geophys. Res, 66, 1407

Bonaccini D., Allaert E., Araujo C., Brunetto E., Buzzoni B., Comin M., Cullum M., Davies R., Dichirico C., Dierickx P., Dimmler M., Duchateau M., Egedal C., Hackenberg W., Hippler S., Kellner S., van Kesteren A., Koch F., Neumann U., Ott T., Quattri M., Quentin J., Rabien S., Tamai R., Tapia M., Tarenghi M., 2002, "'The VLT laser Guide Star Facility"”, in Wizinowich P. L., Bonaccini D. (eds.), Adaptive Optical System Technologies II No. 4839, p. 381

Bowman M., Gibson A., Sandford M., 1969, Nature, 221, 456

Bufton J. L., Minott P., Fitzmaurice M., Titterton P., 1972, "Measurements of Turbulence Profiles in the Troposphere", J. Opt. Soc. Am., 62, 1068

Clemesha B., Kirchhoff W., Simonich D., Takahashi H., 1978, "Evidence of an extra-terrestrial source for the mesospheric sodium layer", Geophys. Res. Lett., 5, 873

Contos A. R., Wizinowich P. L., Hartman S. K., Le Mignant D., Neyman C. R., Stomski P. J., Summers D., 2002, "Laser guide star adaptive optics at the Keck Observatory", in Wizinowich P. L., Bonaccini D. (eds.), Adaptive Optical System Technologies II No. 4839, p. 370

Davies R., Hippler S., Hackenberg W., Ott T., Butler D., Kasper M., Quirrenbach A., 2000, "The ALFA laser guide star: operation and results", ExpA, 10, 103 
Dicke R. H., 1975, "Phase-contrast detection of telescope seeing and their correction", Astron. J., 198, 605

Ellerbroek B. L., 1994, "First-order performance evaluation of adaptiveoptics systems for atmospheric-turbulence compensation in extended-fieldof-view astronomical telescopes", J. Opt. Soc. Am. A, 11, 783

Ellerbroek B. L., Rigaut F., Bauman B., Boyer C., Browne S., Buchroeder R., Catone J., Clark P., l'Orgeville C., Gavel D., Hunten G. H. M. R., James E., Kibblewhite E., McKinnie I., Murray J., Rabaut D., Saddlemeyer L., Sebag J., Stillburn J., Telle J., Véran J.-P., 2002, "MCAO for GeminiSouth", in Wizinowich P. L., Bonaccini D. (eds.), Adaptive Optical System Technologies II No. 4839, p. in press

Esposito S., Ragazzoni R., Riccardi A., O’Sullivan C., Ageorges N., Redfern M., Davies R., 2000, "Absolute tilt from a laser guide star: a first experiment", ExpA, 10, 135

Ewart P., 1985, "A modeless, variable bandwidth, tuneable laser", Optics Com., 55, 124

Filippenko A. V., 1982, "The importance of atmospheric differential refraction in spectrophotometry", Pub. Astron. Soc. Pacific, 94, 715

Foy R., Boucher Y., Fleury B., Grynberg G., McCullough P. R., Migus A., Tallon M., 1992, "ATLAS status report and tilt sensing using multicolour laser reference star", in M.-H. U. (ed.), Progress in telescope and instrumentation technologies, ESO conferences No. 42, ESO, Garching, Germany, p. 437

Foy R., Labeyrie A., 1985, "Feasibility of adaptive telescope with laser probe", AA, 152, L29

Foy R., Migus A., Biraben F., Grynberg G., McCullough P. R., Tallon M., 1995, "The polychromatic artificial sodium star: a new concept for correcting the atmospheric tilt", Astron. Astrophys. Suppl. Ser., 111, 569

Foy R., Tallon M., Tallon-Bosc I., Thiébaut E., Vaillant J., Foy F.-C., Robert D., Friedman H., Biraben F., Grynberg G., Gex J.-P., Mens A., Migus A., Weulersse J.-M., Butler D. J., 2000, "Photometric observations of a polychromatic laser guide star", J. Opt. Soc. Am. A, 17, 2236 
Fried D., 1979, "Angular dependence of atmospheric turbulence effect in speckle interferometry", Opt. Acta, 26, 597

Fried D., 1995, "Focus anisoplanatism in the limit of infinitely many artificial-guide-star reference spots", J. Opt. Soc. Am. A, 12, 939

Fried D., Belscher J., 1994, "Analysis of fundamental limits to artificialguide-star adaptive-optics-system performance for astronomical imaging", JOSAA, 11, 277

Fried D. L., 1965, "Statistics of a geometric representation of wavefront distorsion", J. Opt. Soc. Am., 55, 1427

Froc G., Rosencher E., Atal-Trétout B., Michau V., 2000, "Photon return analysis of a polychromatic laser guide star", Optics Com., 178, 405

Fugate R. Q., Ellerbroek B., Higgings C., Jelonek M., Lange W., Slavin A., Wild W., Wynia J., Spinhirne J., Boeke B., Ruane R., Moroney J., Oliker M., Swindle D., Cleis R., 1994, "Two generations of laser-guide-star adaptive-optics experiments at the Starfire Optical Range", J. Opt. Soc. Am. A, 11, 310

Fugate R. Q., Fried D. L., Amer G. A., Boek B. R., Browne S. L., Roberts P., Ruane R. E., Tyler G. A., Wopat L. M., 1991, "Measurement of atmospheric wavefront distorsion using scattered light from a laser-guide-star", Nature, 353,144

Gardner C., Voelz D., Sechrist C., Segal A., 1986, "Lidar studies of the nighttime sodium layer over Urbana, Illinois. I - Seasonal and nocturnal variations", J. Geophys. Res, 91, 13659

Gastineau C., 1995, Ph.D. thesis, Université Paris-XII, Orsay, France

Gavel D., Gates E., Max C., Olivier S., Bauman B., Pennington D., Macintosh B., Patience J., Brown C., Danforth P., Hurd R., Severson S., Lloyd J., 2002, "Recent Science and Engineering Results with the Laser Guide Star Adaptive Optics System at Lick Observatory", in Wizinowich P. L., Bonaccini D. (eds.), Adaptive Optical System Technologies II No. 4839, p. 354 
Georges III J. A., Mallik P., Stalcup T., Angel J. R. P., Sarlot R., 2002, "'Design and Testing of a Dynamic Refocus System for Rayleigh Laser Beacons"", in Wizinowich P. L., Bonaccini D. (eds.), Adaptive Optical System Technologies II No. 4839, p. 473

Hackenberg W. K., Bonaccini D., Werner D., 2002, "Fiber Raman laser development for multiple sodium laser guide star adaptive optics", in Wizinowich P. L., Bonaccini D. (eds.), Adaptive Optical System Technologies II No. 4839 , p. 421

Happer W., MacDonald G., Max C. E., Dyson F., 1994, "Atmosphericturbulence compensation by resonant optical backscattering from the sodium layer in the upper atmosphere", J. Opt. Soc. Am. A, 11, 263

Humphreys R. A., Bradley L. C., Herrmann J., 1992, "Sodium-layer synthetic beacons for adaptive optics", Lincoln Lab. J., 5, 45

Jegou J.-P., Granier C., Chanin M.-L., Megie G., 1985a, "General theory of the alkali metals present in the earth's upper atmosphere. I - Flux model: Chemical and dynamical processes", Ann. Geophys., 3, 163

Jegou J.-P., Granier C., Chanin M.-L., Megie G., 1985b, "General theory of the alkali metals present in the earth's upper atmosphere. II - Seasonal and meridional variations", Ann. Geophys., 3, 299

Jelonek M. P., Fugate R. Q., Lange W. J., Slavin A. C., Ruane R. E., Cleis R. A., 1994, "Characterization of artificial guide stars generated in the mesospheric layer with a sum-frequency laser", J. Opt. Soc. Am. A, 11, 806

Jeys T. H., Brailove A. A., Mooradian A., 1989, "Sum-frequency generation of sodium resonance radiation", Applied Optics, 28, 2588

Jeys T. H., Heinrichs R. M., Wall K. F., Korn J., Hotaling T. C., Kibblewhite E., 1992, "Observation of optical pumping of mesospheric sodium", Optics L., 17, 1143

Johnston D. C., Welsh B. M., 1994, "Analysis of multiconjugate adaptive optics", J. Opt. Soc. Am. A, 11, 394 
Kasper M., Looze D., Hippler S., Herbst T., Glindemann A., Ott T., Wirth A., 2000, "ALFA: adaptive optics for the Calar Alto observatory - optics, control and performance", Experimental Astronomy, 10, 49

Kibblewhite E., 2000, "The physics of the sodium atom", in Ageorges N., Dainty C. (eds.), Laser Guide Star Adaptive Optics for Astronomy, NATO ASI No. 551, Kluwer Academic Publishers, Dordrecht, p. 51

Kibblewhite E. J., Chun M. R., Larkin J. E., Scor V., Shi F., Smutko M. F., Wild W. J., 1998, "Performance of ChAOS on the Apache Point Observatory's 3.5-m telescope", in Proc. SPIE Vol. 3353, p. 60-71, Adaptive Optical System Technologies, Domenico Bonaccini; Robert K. Tyson; Eds., p. 60

Kolmogorov A., 1961, "The local structure of turbulence in incompressible viscous fluid for very large Reynolds' [sic] numbers", in Friedlander S. K., Topper L. (eds.), Turbulence, Classics Papers on Statistical Theory. Interscience, New York, p. 151

Labeyrie A., 1970, "Attainment of diffraction limited resolution in large telescopes by Fourier analysing speckle patterns in star images", Astron. Astrophys., 6, 85

Laubscher M., Segonds P., Pique J.-P., 2000, "High resolution cw laser beam preamplified in a multipass cavity pumped by a $\mathrm{CuHBr}$ (HyBrID) laser", Optics Com., 184, 425

Le Louarn M., Foy R., Hubin N., Tallon M., 1998, "Laser guide star for 3.6m and $8 \mathrm{~m}$ telescopes: Performances and astrophysical implications", Mon. Not. R. Astron. Soc, 295, 756

Le Louarn M., Tallon M., 2000, "3D mapping of turbulence: theory", in SPIE, Vol. 4007, Wizinowich P. L. (ed.), Adaptive Optical Systems Technology, p. 1066

Le Louarn M., Tallon M., 2002, "Analysis of the modes and behaviour of a Multiconjugate Adaptive Optics system", JOSAA, 19, 912

Lloyd-Hart M., Angel J. R. P., Jacobsen B., Wittman D., Dekany R., McCarthy D., Kibblewhite E., Wild W., Carter B., Beletic J., 1995, “Adaptive optics experiments using sodium laser guide stars", Astrophys. J., 439, 455 
Max C., Olivier S., Friedman H., An K., Avicola K., Beeman B., Bissinger H., Brase J., Erbert G., Gavel D., Kanz K., Liu M., Macintosh B., Neeb K., Patience J., Waltjen K., 1997, "Image improvement from a sodium-layer guide star adaptive optics", Science, 277, 1649

Megie G., Blamont J., 1977, "Laser sounding of atmospheric sodium interpretation in terms of global atmospheric parameters", Planet. Space Sci., 25, 1093

Michaille L., Canas T. A., Dainty C., Maxwell J., Gregory T. S., Quartel J. C., Reavell F. C., Wilson R. W., Wooder N. J., 2000, "Monitoring the variation of mesospheric sodium density at La Palma", in SPIE, Vol. 4007, Wizinowich P. L. (ed.), Adaptive Optical Systems Technology, p. 349

Olivier S. S., Gavel D. T., Friedman H. W., Max C. E., An J. R., Avicola K., Bauman B., Brase J. M., Campbell E., Carrano C., Cooke J., Freeze G., Gates E., Kanz V., T K., C, Macintosh B., Newman M., Pierce E., Waltjen K., Watson J., 1999, "Improved performances of the laser guide star adaptive optics at Lick Observatory", in SPIE, Vol. 3762, Tyson R. K., Fugate R. Q. (eds.), Adaptive optics systems and technology, p. 2

O'Sullivan C., Redfern M., Ageorges N., Holstenberg H.-C., Hackenberg W., Ott T., Rabien S., Davies R., Eckart A., 2000, "Short timescale variability of the mesospheric sodium layer", ExpA, 10, 147

Papen G. C., Gardner C. S., Yu J., 1996, "Characterization of the mesospheric sodium layer", in OSA, Vol. 13, OSA conf. on Adaptive Optics. OSA, p. 96

Parenti R. R., Sasiela R. J., 1994, "Laser-guide-star systems for astronomical applications", JOSAA, 11, 288

Pennington D. M., Brown C. G., Danforth P. M., Jones H. E., Max C. E., Chin J., Lewis H., Medeiros D., Nance C. E., Stomski P. J., Jr. , Summers D., Wizinowich P. L., 2002a, "Performance and status of the Keck Observatory guide star laser system", in Wizinowich P. L., Bonaccini D. (eds.), Adaptive Optical System Technologies II No. 4839, p. in press

Pennington D. M., Hackenberg W. K., Beach R. J., Bonaccini D., Drobshoff A., Ebbers C. A., Liao Z. M., Payne S. A., Taylor L., 2002b, "Compact fiber 
laser approach to generating 589-nm laser guide stars", in Wizinowich P. L., Bonaccini D. (eds.), Adaptive Optical System Technologies II No. 4839, p. in press

Pentland G., Blanco D. R., Rebeske K., Winrow E. G., d'Orgeville C., 2002, "Preliminary design of a laser launch telescope for Gemini", in Wizinowich P. L., Bonaccini D. (eds.), Adaptive Optical System Technologies II No. 4839, p. 429

Pilkington J., 1987, "Artificial guide stars for adaptive imaging", Nature, 330,116

Pique J.-P., Farinotti S., 2003a, "" An efficient modeless laser for a mesospheric sodium laser guide star"", in , CLEO Europe, p. in press

Pique J.-P., Farinotti S., 2003b, "An efficient modeless laser for a mesospheric sodium laser guide star", J. Opt. Soc. Am. A, submitted

Primmerman C. A., Murphy D. V., Page D. A., Zollars B. G., Barclay H. T., 1991, "Compensation of atmospheric optical distortions using a synthetic beacon", Nature, 353, 141

Rabien S., Davies R., Ott T., li ans S[tephan] Hippler J., Neumann U., 2002, "Design of PARSEC, the VLT laser", in Wizinowich P. L., Bonaccini D. (eds.), Adaptive Optical System Technologies II No. 4839, p. 393

Rabien S., Ott T., Hackenberg W., Eckart A., Davies R., Kasper M., Quirrenbach A., 2000, "The ALFA laser and analysis tools", Experimental Astronomy, 10, 75

Ragazzoni R., 1997, "Robust tilt determination from Laser Guide Star using a combination of different techniques", Astron. Astrophys., 319, L9

Ragazzoni R., Esposito S., Marchetti E., 1995, "Auxiliary telescopes for the absolute tip-tilt determination of a laser guide star", Mon. Not. R. Astron. Soc, 276, L76

Ragazzoni R., Marchetti E., Rigaut F., 1999, "Modal tomography for adaptive optic", Astron. Astrophys., 342, L53

Richter E., Sechrist C., 1979a, "A cluster ion chemistry for the mesospheric sodium layer", J. atmos. terr. Phys., 41, 579 
Richter E. S., Sechrist C. F., 1979b, "A meteor ablation-cluster ion atmospheric sodium theory", Geophys. Res. Lett., 6, 183

Rigaut F., Gendron E., 1992, "Laser guide star in adaptive optics: the tilt determination problem", Astron. Astrophys., 261, 677

Roddier F., 1981, "The effects of atmospheric turbulence in optical astronomy", Progress in Optics, XXI, 281

Rousset G., Lacombe F., andÉric Gendron P. P., Arsenault R., Kern P., Rabaud D., Madec P.-Y., Hubin N., Zins G., Stadler E., Charton J., Gigan P., Feautrier P., 2000, "Status of the VLT Nasmyth adaptive optics system (NAOS)", in SPIE, Vol. 4007, Wizinowich P. L. (ed.), Adaptive Optical Systems Technology, p. 72

Rousset G., Lacombe F., Puget P., Hubin N., Gendron E., Fusco T., Arsenault R., Charton J., Feautrier P., Gigan P., Kern P., Lagrange A.-M., Madec P.-Y., Mouillet D., Rabaud D., Rabou P., Stadler E., Zins G., 2002, "'NAOS, the first AO system of the VLT: on-sky performances"”, in Wizinowich P. L., Bonaccini D. (eds.), Adaptive Optical System Technologies II No. 4839, p. 140

Rutten R. G. M., Clarck P., Myers R. M., Wilson R. W., Bingham R. G., Emsellem É., Gregory T., Humphreys R. A., Knapen J. H., Moretto G., Morris S. L., Talbot G., 2002, "'Facility class Rayleigh beacon AO system for the 4.2m William Hershel Telescope" ", in Wizinowich P. L., Bonaccini D. (eds.), Adaptive Optical System Technologies II No. 4839, p. 360

Sandler D. G., Stahl S., Angel J., Lloyd-Hart M., McCarthy D., 1994, "Adaptive optics for diffraction-limited infrared imaging with 8-m telescopes", JOSAA, 11, 925

Schöck M., Foy R., Tallon M., Noethe L., Pique J.-P., 2002, "Performance Analysis of Polychromatic Laser Guide Stars Used for Wavefront Tilt Sensing", MNRAS, 337, 910

Séchaud M., Hubin N., Brixon L., Jalin R., Foy R., Tallon M., 1988, "Laser Backscattered Reference Star for Atmospheric Wavefront Disturbances Measurement", in Ulrich M.-H. (ed.), Very Large Telescopes and their Instrumentation, ESO/NOAO Conferences No. 30, ESO, Garching, Germany, p. 705 
Slipher V., 1929, PASP, 41, 262

Stone R. C., 1989, "A comparison of digital centering algorithms", AJ, 97, 1227

Swider W., 1985, "Enhanced seasonal variations for chemical rates with inverse temperature dependencies - Application to seasonal abundance of mesospheric sodium", Geophys. Res. Lett., 12, 589

Tallon M., 1997, private communication

Tallon M., Foy R., 1990, "Adaptive optics with laser probe: isoplanatism and cone effect", Astron. Astrophys., 235, 549

Tallon M., Foy R., Blazit A., 1988, "Real time wavefront sensing at CFH telescope", in Ulrich M.-H. (ed.), Very Large Telescopes and their Instrumentation, ESO/NOAO Conferences No. 30, ESO, Garching, Germany, p. 743

Tallon M., Foy R., Vernin J., 1992a, "3-D wavefront sensing for multiconjugate adaptive optics", in M.-H. U. (ed.), Progress in telescope and instrumentation technologies, ESO conferences No. 42, ESO, Garching, Germany, p. 517

Tallon M., Foy R., Vernin J., 1992b, "Wide field adaptive optics using an array of laser guide stars", in Fugate R. Q. (ed.), Laser guide star adaptive optics workshop, SOR/Phillips Lab., Albuquerque (NM), p. 555

Taylor G. I., 1938, "The spectrum of turbulence", Proc. Roy. Soc.London, A164, 476

Telle J. M., Milonni P., Fugate R., 2000, "Update on 589-nm sodium guide star pump laser requirements", in Wizinowich P. L. (ed.), Adaptive Optical Systems Technology, SPIE, p. 252

Thompson L. A., Castle R. M., W.Teare S., McCullough P. R., Crawford S. L., 1998, "UnISIS: a laser-guided adaptive optics system for the Mt. Wilson 2.5-m telescope", in SPIE, Vol. 3353, Bonaccini D., Tyson R. K. (eds.), Adaptive Optical System Technologies, p. 282 
Thompson L. A., Gardner C. A., 1987, "Experiment on laser guide stars at Mauna Kea Observatory for adaptive imaging in astronomy", Nature, 328, 229

Thompson L. A., Xiong Y.-H., 1995, "Laser beacon system for the UnISIS adaptive optics system at the Mount Wilson 2.5-m telescope", in SPIE, Vol. 2534, p. 38

Tokovinin A., Le Louarn M., Sarazin M., 2000, "Isoplanatic angle in multiconjugate adaptive optics system", JOSAA, 17, 1819

Tyler G. A., 1994, "Bandwidth considerations for tracking through turbulence with 8-m telescopes", JOSAA, 11, 358

T.Young A., 1970, "Aperture filtering and saturation of scintillation", J. Opt. Soc. Am., 60, 248

Vaillant J., Thiébaut É., Tallon M., 2000, "ELPOA: Data processing of chromatic differences of the tilt measured with a polychromatic laser guide star", in SPIE, Vol. 4007, Wizinowich P. L. (ed.), Adaptive Optical Systems Technology, p. 308

Vernin J., Azouit M., 1983, "Traitement d'image adapté au speckle atmosphérique. II. Analyse multidimensionnelle appliquée au diagnostic à distance de la turbulence", J. Optics (Paris), 14, 131

Vernin J., Mũnoz-Tuñón C., 1992, "Optical seeing at La Palma Observatory. I. General guidelines and preliminary results at the Nordic Optical Telescope", Astron. Astrophys., 257, 811

Vernin J., Mũnoz-Tuñón C., 1994, "Optical seeing at La Palma Observatory. II. Intensive site testing campaign at the Nordic Optical Telescope", Astron. Astrophys., 284, 311

Viard E., Hubin N. H., Le Louarn M., 2000, "Adaptive optics with four laser guide star: cone effect correction on large telescopes", in SPIE, Vol. 4007, Wizinowich P. L. (ed.), Adaptive Optical Systems Technology, p. 106

von Zahn U., Hansen T., 1988, "Sudden neutral sodium layers: a strong link to sporadic E layers", J. atmos. terr. Phys., 50, 93 
Wizinowich P., Acton D. S., Gregory T., Stomski P., An J., Avicola K., Brase J. M., Friedman H. W., Gavel D. T., Max C. E., 1998, "Status of the W.M. Keck Observatory adaptive optics facility", in SPIE, Vol. 3353, Bonaccini D., Tyson R. K. (eds.), Adaptive optical system technologies, p. 568

W.Milonni P., Fugate R., Telle J. M., 1998, "Theory of continuous-wave excitation of the sodium beacon", J. Opt. Soc. Am. A, 16, 2555

Wuerker R. F., 1997, "Sporadic sodium layer formation during an auroral storm", in N. H. (ed.), Laser technology for laser guide star adaptive optics astronomy, ESO Workshops, ESO, Garching, Germany, p. 16

Zollars B., 1992, "Atmospheric-turbulence compensation experiments using synthetic beacons", Lincoln Lab. J., 5, 67 